\title{
Minor ions in the solar wind
}

\author{
Peter Bochsler
}

Received: 4 May 2006 / Published online: 8 November 2006

(C) Springer-Verlag 2006

\begin{abstract}
Ions heavier than ${ }^{4} \mathrm{He}$ are treated as "minors" in the solar wind. This is justified for many applications since minor ions have no significant influence on the dynamics of the interplanetary plasma. However, minor ions carry information on many aspects of the formation, on the acceleration and on the transfer of solar plasma from the corona into the interplanetary space. This review concentrates on various aspects of minor ions as diagnostic tracers. The elemental abundance patterns of the solar wind are shaped in the chromosphere and in the lower transition region by processes, which are not fully understood at this moment. Despite this lack of detailed understanding, observed abundance patterns have been classified and are now commonly used to characterize the sources, and to trace back solar-wind flows to their origins in the solar atmosphere. Furthermore, the solar wind is the most important source of information for solar isotopic abundances and for solar abundances of volatile elements. In order to fully exploit this information, a comprehensive understanding of elemental and isotopic fractionation processes is required. We provide observational clues to distinguish different processes at work.
\end{abstract}

Keywords Solar Wind · Sun · Composition

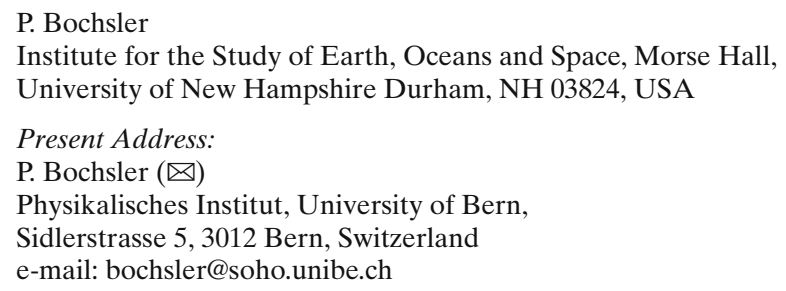




\section{Introduction}

Particle mass, nuclear charge and state of ionization, $A, Z$, and $Q$, are quantities which characterize a minor ion in the solar wind. These parameters, together with the three velocity components $v_{x}, v_{y}, v_{z}$ suffice to describe a single particle at a given point and time. How is this information to be used?

There are two distinct branches of interest, each with wide ramifications and with important cross-links between each other. The first is related to the last four parameters, i.e., to kinetic properties of the minor ion and its charge state: they describe the actual state of the particle, and in the context of its ambient environment, they can provide relevant information on the dynamic state of the ambient plasma and on the interaction of a particle with its surroundings, i.e., on its collisions with field particles, its interaction with the magnetic field, wave-particle interactions, etc. In this sense minor ions have been used as "hydrological tracers," that is particles which do not influence the flow itself but serve as diagnostics for processes occurring in the flow. The most prominent application, which comes to mind, is the use of charge-state distributions to investigate coronal temperatures, coronal temperature gradients and density gradients. This covers the physical aspects of interest in minor species.

The chemical interest concerns the parameters $A$ and $Z$, i.e., the composition of minor species in the solar wind. Again, as will be outlined in more detail in the following, the abundance pattern of minor species in relation to solar photospheric abundances can tell us something about the solar-wind feeding process. For some elements, especially those with high excitation energies (and high ionization potentials) such as the noble gases and the volatile elements in general, the solar-wind abundances provide important information on solar abundances. ${ }^{1}$

An even more interesting application is the determination of isotopic abundances from the solar wind. In this case the solar wind provides information on the Sun, which is not accessible through optical observations or through other means.

The two foci of interest should not be treated independently because they are closely interlinked. Understanding the interaction of minor species with the surrounding magnetized plasma is crucial for the understanding of the relation between solar-wind abundances and photospheric abundances. Conversely, if the abundance of an element or its isotopic composition at the coronal base and in the solar-wind is known with good precision, it is possible to draw useful inferences about the dynamics of the corona and the interplanetary plasma.

\footnotetext{
1 Classically, speaking about solar elemental abundances, one refers to abundances determined from the depths and shapes of lines observed in the photospheric spectrum. Elements with high excitation energies and ionization potentials do not usually have lines in the photospheric spectrum that are suitable for an abundance determination. Chemically, such elements are highly volatile and meteoritic or even cometary abundances do not allow reliable inferences for solar abundances. Coronal abundances of such elements can, however, be obtained from observations in the extreme ultraviolet and X-ray spectral ranges observed outside the terrestrial atmosphere.
} 
Almost simultaneously with the first experimental confirmation of a continuously blowing solar wind (Gringauz et al. 1960, 1961) it was discovered that the solar wind contained also elements other than hydrogen: Neugebauer and Snyder $(1962,1966)$ used a combination of a simple E/Q-analyzer and a Faraday cup on the Mariner 2 spacecraft to investigate the energy per charge distribution of the solar-wind particles. Scanning in coarse steps through a wide range of proton energies they found that ${ }^{4} \mathrm{He}^{++}$was a sizeable constituent. Despite the poor resolution of the E/Q-spectra obtained from the Mariner experiment, they derived a helium to hydrogen flux ratio of 0.046 , remarkably compatible with present-day determinations of the helium abundance in the solar wind, although these authors were forced to attribute a rather high uncertainty to their first measurement. Nevertheless, the uncertainty was small enough to let the authors realize that the abundance of helium in the solar wind was different from its putative solar abundance [see Neugebauer (1997), Hufbauer (1991), Parker (2001) for a detailed account of these early discoveries].

For a long time, minor ions in the solar wind have mainly been a topic of interest for the in situ community, i.e., they have been investigated with in situ instruments of increasing complexity - going from electrostatic energy/charge analyzers (Bame et al. 1968, Rosenbauer et al. 1977), to combined Wien Filters with electrostatic energy/charge analyzers (Coplan et al. 1978) to sophisticated time-of-flight mass-spectrometers (Gloeckler et al. 1983, Hovestadt et al. 1995). More recently, it has become possible to study minor species and their kinetic properties in the corona by remote-sensing techniques, notably with the UVCS and SUMER instruments on SOHO (Kohl et al. 1995, Wilhelm et al. 1995, Cranmer et al. 1999b, Kohl et al. 2006).

Soon after it was realized that helium was generally depleted in the solar wind compared to solar abundances, oxygen ions were detected by the Los Alamos Group (Bame et al. 1968). This confirmed the view that apart from the notorious underabundance of helium, the other elements were contained in generally solar proportions. Simultaneously, the Los Alamos Group demonstrated that the minor ions carried information on physical properties of the innermost corona far out into the interplanetary space. These observations allowed the first determination of coronal temperatures with in situ techniques (Hundhausen et al. 1968b).

Not much after the experimental confirmation of the existence of the solar wind, Wänke (1965) attributed large amounts of noble gases found in brecciated meteorites to a solar origin. In his paper Wänke hypothesized that solar gases had been implanted with the solar wind into meteoritic grains, possibly in an asteroidal regolith. Eberhardt et al. (1966), analyzing different grain size fractions of the Khor-Temiki meteorite, succeeded to demonstrate that, indeed, these gases were surface correlated and had been implanted with typical solar wind energies into the grains.

This discovery ultimately led Geiss et al. (1972) (see also Geiss et al. 2004) to develop a solar-wind-trapping experiment with an artificial target and to investigate solar-wind noble gases by deploying an aluminum foil on the Moon in the course of the Apollo missions. With the Apollo missions, it was also 
possible to study elemental and isotopic abundances of solar-wind noble gases and some other elements up to xenon by analyzing lunar dust in the laboratory (Eberhardt et al. 1970). Now it became evident that the solar wind contained the full periodic system of elements roughly in solar abundances. All these discoveries opened the two intertwined avenues of research: What can we learn about solar abundances from the solar wind? And, typical thermal speeds of heavy ions at coronal temperatures are far below escape speeds from the solar gravitational field; but then, how is it possible that heavy species are efficiently incorporated into the solar wind?

These questions will be addressed in the following. One of the purposes of this review is to demonstrate the wealth and diversity of physical topics to be found in this field, once one accepts the fact that the solar wind does not only consist of protons and electrons. We begin with a presentation of some basics about the solar corona in Sect. 2.

\section{Minor species in the solar corona}

In order to understand the observations of kinetic properties and abundance variations of minor species, we discuss some salient points in the physics of the solar corona. As indicated before, we consider minor species to be test particles, and we will not enter a detailed discussion of field particles, i.e., protons and electrons. The theory of the field particles in the solar wind that describes the steady hydrodynamic extension of the corona into the interplanetary space has been developed by E.N. Parker in a series of articles (Parker 1958, 1961, 1965).

The Sun heats its corona to a temperature of $1-2$ million degrees K (MK), i.e., protons and electrons reach typical thermal energies $k T=100 \mathrm{eV}$. Hydrogen and helium become fully ionized; species heavier than helium are almost fully ionized, i.e., the dominating oxygen species is $\mathrm{O}^{6+}$. Oxygen, for example, can be brought to this level of ionization in steps of typically $100 \mathrm{eV}$. The transition from $\mathrm{O}^{6+}$ to $\mathrm{O}^{7+}$, however, requires $740 \mathrm{eV}$. Iron is present near the coronal temperature maximum in the form of $\mathrm{Fe}^{10+}$ to $\mathrm{Fe}^{14+}$.

Such elevated charge states can hardly be produced by radiation from the solar disk. The EUV radiation from the solar atmosphere is generally too weak, except in some active regions of limited spatial extent. The corona itself does produce such energetic radiation but it occurs in single spectral lines and, moreover, it is optically thin. The white light coronal brightness is at least six orders of magnitude below the brightness of the solar disk Bird and Edenhofer (1990). Electron collisions play an important role in achieving these high ionization states, hence, charge states of minor ions bear information on electron temperatures near the coronal temperature maximum.

The gravitational energy of a proton near the coronal temperature maximum is $-1660 \mathrm{eV}$; for an oxygen ion it amounts to $-27 \mathrm{keV}$. Comparing these numbers with the thermal energies in the still collision-dominated regime, shows that heavy species remain far below escape speed. Hence, some interaction with lighter particles or extra heating of heavy species is necessary to 
incorporate them into the solar-wind flow. Typical densities near temperature maximum are $10^{13}$ particles per $\mathrm{m}^{3}$. The collision time in a plasma under such conditions is of the order of $100 \mathrm{~s}$, corresponding to a mean free path of $10 \mathrm{Mm}$ at the relevant temperature, which is still an order of magnitude smaller than the density scale height, showing that indeed, heavy particles are collisionally coupled to protons and electrons near temperature maximum. As particles move beyond the coronal temperature maximum, electron densities continue to decrease and the coupling with coronal electrons diminishes accordingly. We will exploit this in Sect. 4.1 when discussing the determination of coronal temperature gradients by means of charge-state abundances of minor ions.

Low in the chromosphere, typical particle densities are of the order of $10^{18}$ particles per $\mathrm{m}^{3}$. Putting this into relation to a typical solar-wind flux, one obtains a flow speed of $0.2 \mathrm{~m} / \mathrm{s}$ in the chromosphere and a typical transfer time to the site of the coronal temperature maximum at a fraction of $1 \mathrm{R}_{\odot}$ above the solar surface, of the order of several weeks. Considering the magnitude of thermal velocities and even more the vigorous dynamics of phenomena in the solar atmosphere as evidenced from TRACE or SOHO movies (see: http://sohowww.nascom.nasa.gov/data/realtime/mpeg or http://race.lmsal.com/ Public/Gallery/Movies), it is clear that these numbers represent statistical averages and do not imply motion in a steady state. In a statistical description one might say that chances of an average particle to be fed from the solar atmosphere into the corona at a given moment, are extremely small. Once, however, such a particle has reached the corona it will continue its travel into the interplanetary space in a steady supersonic and super-alfvénic flow following the picture delineated by Parker. Beyond this point one expects that also heavy particles are carried into space more or less indiscriminately. On the other hand, the order of magnitude of the collision times indicates that the incorporation of heavy ions through Coulomb collisions with protons is not self-evident. We will discuss the effect of Coulomb collisions in more detail, especially keeping in mind that the solar-wind flow is highly structured and that high-density structures might play an important role in feeding heavy species into the solar wind.

Heating and accelerating minor ions in the solar wind is intricately linked to the general problem of coronal heating. In fact, minor ions have provided substantially deeper insight into the coronal heating mechanisms (see the articles of Cranmer et al. (1999a,b) and the review by Cranmer (2002) for a detailed account). In order to accelerate solar-wind ions including minor species, heating of the supersonic flow is necessary. Apparently this heating mechanism is most efficient when the kinetic properties of protons and ions are affected differently, specifically if $T_{\perp}$ is preferentially enhanced over $T_{\|}$, where the subscripts indicate the direction of the thermal spread relative to the ambient magnetic field.

All theoretical models agree that the import of energy into the flow arises from some type of MHD fluctuations, waves or turbulence. The source and the propagation mechanisms of these fluctuations have not been clearly identified, ultimately they must be related to the convective motions in the outer 
convection zone of the Sun (OCZ). Possibly, they are due to small-scale reconnection events in the solar atmosphere. In principle, understanding the origin of these magnetic disturbances is not so important to explain the heating and acceleration of heavy species. However, it is very important to understand how these disturbances continuously feed heavy species into the transition region and into the corona. The problem inherent in linear theories of coronal waveheating is that either - due to the small pressure- and temperature-scale-heights in the solar atmosphere, waves are damped very rapidly and will not reach the regions, where heat addition is required - and/or, that these waves when they finally reach the location where heating is needed, will not dissipate and, hence, not deposit energy into the ambient plasma. The solution lies probably in finding a self-consistent theory of MHD turbulence cascading energy from large structures into fine structures and ultimately into individual particles. MHD waves can exert pressure on ions and accelerate them through pressure gradients in a non-resonant and non-discriminating wave-particle interaction. This has been explored by several authors at different occasions, e.g., (McKenzie et al. 1979, Isenberg and Hollweg 1982, McKenzie 1994).

The alternative to non-resonant wave heating and acceleration of minor ions is ion-cyclotron heating - preferentially in the plane perpendicular to the direction of the magnetic field - combined with acceleration by the mirror force exerted by the diverging field. This mechanism has increasingly gained popularity since remote sensing with the $\mathrm{SOHO}$ spacecraft brought clear evidence for enhanced $\mathrm{O}^{5+}$-kinetic temperatures, with preferential thermal motion perpendicular to the ambient field (e.g., Kohl et al. 1997, Cranmer 2002)

\section{Observations on dynamics of minor ions}

Figure 1 (from Marsch et al. 1982) is a comprehensive illustration of various situations encountered in low-speed and high-speed solar-wind streams. Lowspeed solar wind (sometimes also named "interstream" solar wind) is thought to originate from the boundaries of closed-loop systems. This type of wind usually remains collision-dominated out to large solar distances. High-speed wind - or more precisely - "coronal-hole-associated" solar wind is dominated by waveparticle interaction producing strong anisotropies in the velocity distributions of all ionic species. It originates from coronal holes.

Panels A and B in Fig. 1 show iso-phase-space density contour plots of typical isotropic distributions with rather low temperatures and high proton densities measured in low-speed solar wind with the Helios spacecraft. As expected for a thermally fully equilibrated plasma, such distributions are representative for collisional regimes, where collision times are smaller than or comparable to the solar-wind expansion time. For the discussion of kinetic properties of minor species, it is useful to consider collisional time scales in some more detail. Spitzer (1956) gives an expression for an equipartition time, within which test particles (subscript $i$ ) adapt their kinetic temperatures to a Maxwellian distribution of field particles characterized with a kinetic temperature $T$ : 

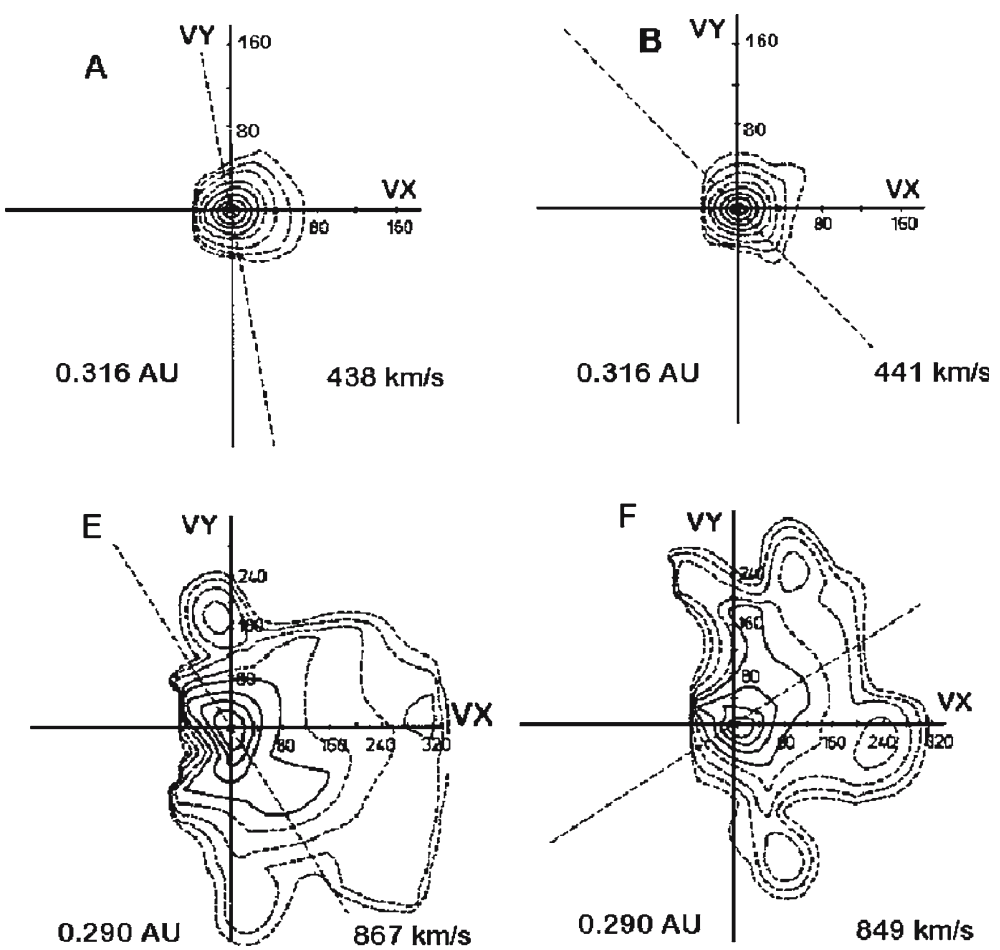

Fig. 1 Cuts of the three-dimensional velocity distributions of helium ions obtained with the Helios spacecraft. The origins of the plots are centered about the core of the drift velocities of helium. The $v_{x}$ axis points in the direction of the bulk velocity vector. The cuts are taken in the plane determined by the $v_{x}$ axis and the magnetic field vector. The direction of the magnetic field vector is indicated by the dashed line (from Marsch et al. (1982))

$$
\begin{aligned}
t_{\text {eq,thermal }} & =: \frac{T_{i}-T}{\mathrm{~d} T / \mathrm{d} t} \\
t_{\mathrm{eq}, \text { thermal }} & =\frac{3 m m_{i} \pi^{3 / 2} \epsilon_{o}^{2}}{2 n Q^{2} Q_{i}^{2} e^{4} \ln \Lambda}\left(\frac{2 k T_{i}}{m_{i}}+\frac{2 k T}{m}\right)^{3 / 2} .
\end{aligned}
$$

$\ln \Lambda$ is the so-called Coulomb logarithm, which takes a value of typically 20 in the solar wind (Spitzer 1956). From the data given by Marsch et al. (1982) one finds a typical equilibration time of $40,000 \mathrm{~s}$ for the situation shown in panels $\mathrm{A}$ and $\mathrm{B}$. The solar-wind expansion-time is usually defined as the time it takes the plasma to travel through a density-scale-height

$$
t_{\exp }=\frac{\mathrm{d} r}{(v \mathrm{~d} \ln n)} .
$$

Since, the density decreases with $1 / r^{2}, t_{\exp }=r / 2 v$. In the case of panels A and $\mathrm{B}$ in Fig. 1 this amounts to 50,000 s, i.e., exceeding somewhat the collision time. 
Livi and Marsch (1987) have shown that a few collisions within a solar-wind expansion time-scale suffice to keep the core of a distribution in thermal equilibrium and isotropic.

Panels $\mathrm{E}$ and $\mathrm{F}$ in Fig. 1 show helium velocity distributions as encountered in high-speed solar wind. Whereas the core of the distribution appears rather isotropic, the surroundings show complex structures, revealing a complicated history of the distribution. In this case, the bulk of the helium distribution was drifting at a relatively high differential speed relative to protons, the speed difference mounting to typically $150 \mathrm{~km} / \mathrm{s}$, i.e., close to the ambient Alfvén speed.

It has been demonstrated in many instances that minor ions travel faster than protons (e.g., Ogilvie 1975). We refer to a comprehensive early summary of the observational evidence by Asbridge et al. (1976) and note in passing that already in this early publication one finds histograms strongly peaked about $\left|v_{\alpha}-v_{\mathrm{p}}\right| \approx 0$, where $\alpha$ and $\mathrm{p}$ refer to helium particles and protons. This phenomenon will be discussed in the following in the context of minor ions speeds.

Hollweg (1974) contributed to the theoretical foundations for explaining the preferential acceleration of helium in the solar wind. Later, several other studies focusing on bulk speeds of heavy ions have been carried out with different instruments on different spacecraft, i.e., with the Ion Composition Instrument (ICI) on ISEE-3 (Ogilvie et al. 1980a, Schmid et al. 1987, Bochsler 1989), with SWICS on Ulysses (von Steiger et al. 1995), and with CELIAS on SOHO (Hefti et al. 1998). As others had shown previously, the work of Hefti et al. (1998) demonstrated that heavy ions tend to travel significantly faster in the fast solar wind, whereas they show no significant deviation from proton speeds in slow solar wind. This is illustrated in Fig. 2.

The comparison of $\mathrm{O}^{6+}$ with protons in the top left panel comes as expected. Oxygen (as is usually the case also for $\mathrm{He}^{++}$travels faster than protons in highspeed winds. The other panels confirm these findings through measurements made with other instruments (Bochsler 1989, Schmid et al. 1987): Si and Fe ions tend to lag somewhat behind oxygen. Tu et al. (2003) made an attempt to explain these observations in numerical simulations, taking into account inward- and outward-propagating waves, and including the second harmonic of ion-cyclotron resonance and subtle differences of different minor species in their cyclotron resonances with various types of waves. It is noteworthy that all these features are less obvious or absent in observations with Ulysses/SWICS (von Steiger et al. 1995). Note that during the observations of von Steiger et al. (1995) Ulysses was traveling at heliocentric distances between 5.3 and 4.5 AU, whereas the other observations were carried out on halo orbits around the Euler-Lagrange point L1 in the Earth-Sun system, around 0.99 AU from the Sun.

The discrepancy in observations at different sites confirms the fact that waveparticle interaction continues to shape velocity distributions of minor species beyond $1 \mathrm{AU}$. In addition, stream-stream interactions must have considerably smeared out features by the time they arrived at Ulysses, whereas they are still clearly visible at $1 \mathrm{AU}$. During a period of a full year the helium speed hardly 

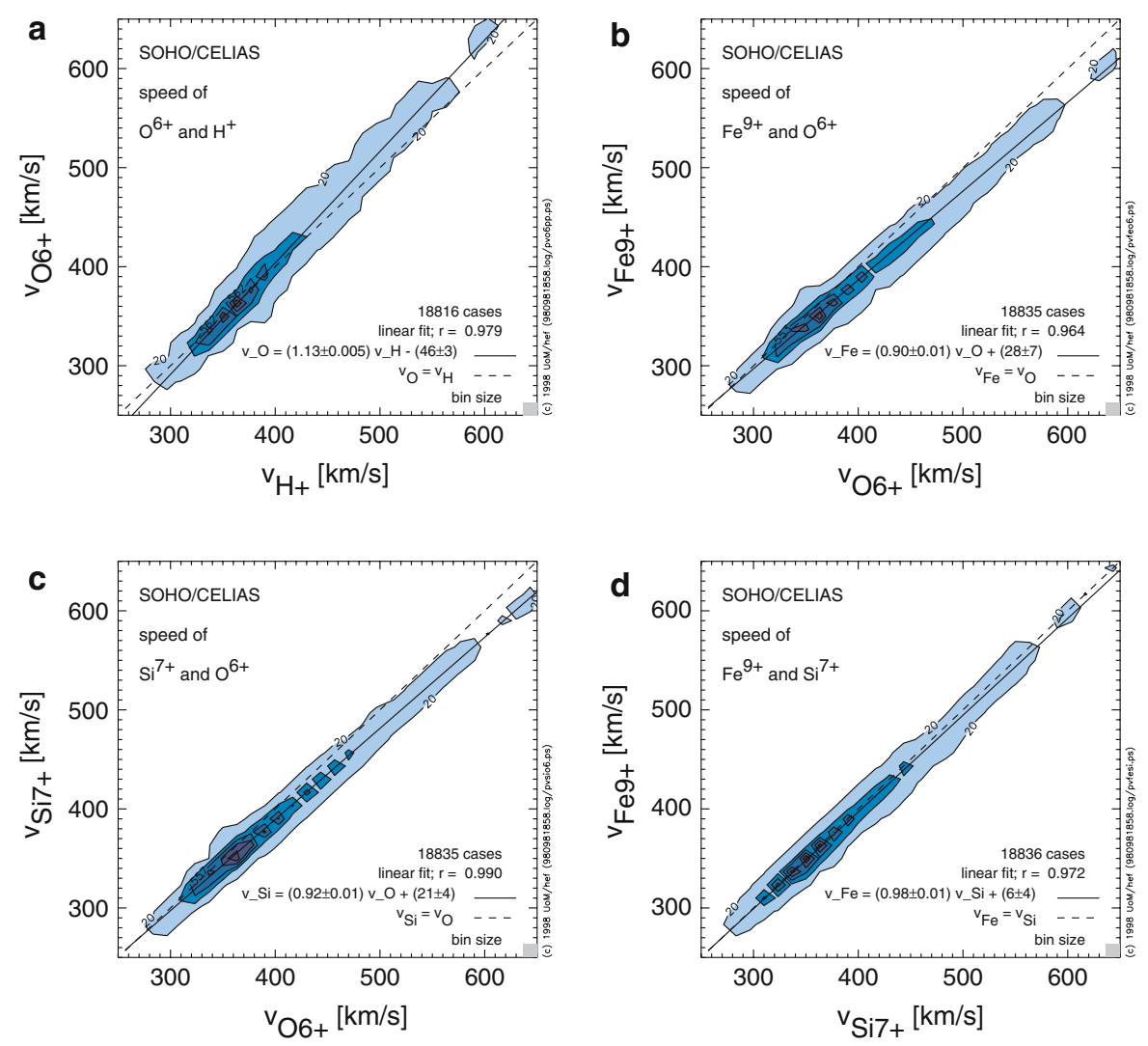

Fig. 2 Histograms of bulk velocities of several heavy species. The dashed lines show the locus of two species that have equal drift speeds. It is evident that in the case of low speeds the observations match this line, whereas in the case of high-speed solar wind significant differences of the order of $20-40 \mathrm{~km} / \mathrm{s}$ are observed (from Hefti et al. 1998)

dropped to values below $400 \mathrm{~km} / \mathrm{s}$ at the Ulysses orbit, despite the fact that periods with the typical compositional characteristics of low-speed solar wind have been included in the observations. This demonstrates impressively that kinetic properties of minor species in the solar wind are much less conservative properties than compositional signatures.

Differential streaming of different species can create a beam instability even in the absence of collisions, which tends to redistribute kinetic and thermal energy among different species. This has been discussed by McKenzie (1994) and verified more recently experimentally with Ulysses/SWOOPS observations by Reisenfeld et al. (2001). As the solar-wind plasma moves outward, the ambient Alfvén velocity decreases and helium ions, which travel at speeds exceeding the Alfvén speed in the frame of the bulk solar wind, will readjust their drift velocity, converting kinetic energy into heat as a consequence of the onset of a magnetosonic instability. This mechanism might also be involved in readapting 
differential streaming of species heavier than helium, thereby being subject to different growth rates of the instability. This needs to be investigated in future work.

A detailed analysis of velocities of heavy species over a more extended time series obtained by ISEE-3/ICI (Bochsler 1989) revealed a conspicuous histogram of velocity differences $v_{\mathrm{Si}}-v_{\alpha}$. ISEE-3/ICI with its combined Wien-Filter and electrostatic energy analyzer provided particularly well resolved velocity distributions of heavier species. In this study, the different charge states of silicon were not resolved (they ranged from $\mathrm{Si}^{8+}$ to $\mathrm{Si}^{10+}$, i.e., $3.0 \leq M / Q \leq 3.6$ ). The pointed peak near the bin of zero velocity differences in Fig. 3 indicates that zero drift velocity differences are particularly stable situations in the solarwind flow. Consider a plasma parcel with different species traveling through the corona and the interplanetary space under the antagonistic influences of wave-action and Coulomb collisions. Apparently there is an agent, which acts most efficiently to equalize speeds when the bulk speed differences between the different species is smallest. In such a case an initial speed difference between different species will either remain large because equilibration is slow, or, it will rapidly converge towards small speed differences as relaxation times strongly decrease with speed difference.

The relaxation time for two different species with different drift speeds is defined in analogy with (1)

$$
t_{\text {eq,speed }}=: \frac{u_{i}-U}{\mathrm{~d} u_{i} / \mathrm{d} t} .
$$
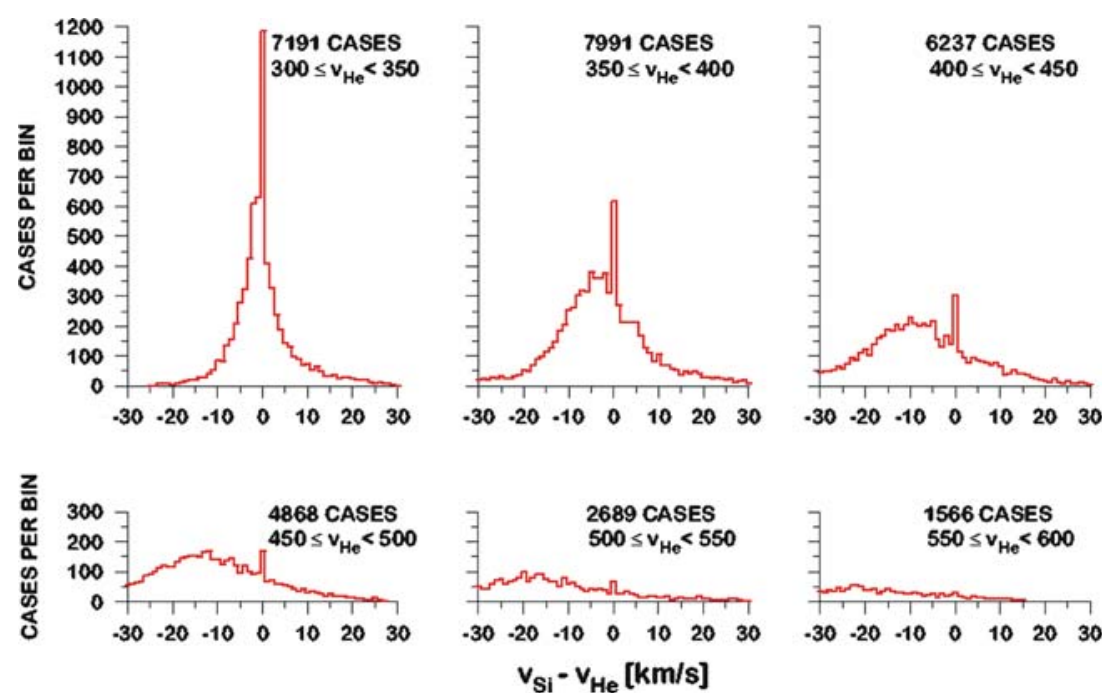

Fig. 3 Histograms of velocity difference between silicon and helium ions observed with the Ion Composition Instrument on ISEE-3 from 1978 through 1982 (Bochsler 1989). Note that the differences widen and tend towards negative values for solar-wind samples with high speeds. Note also the conspicuously pointed peak in low-speed solar-wind regimes 
This characteristic time can readily be derived from the transport equation of minor species, which includes the influence of Coulomb collisions (Geiss et al. 1970, Leutwyler 1999). The speed adjustment time for test particles of species $\mathrm{i}$ $\left(m_{i}, u_{i}, Q_{i}, T_{i}\right)$ streaming in a Maxwellian distribution relative to field particles $(m, U, Q, T)$, having a density $n$ is:

$$
t_{i, \text { eq }, \text { speed }}=\frac{2 \pi \epsilon_{0}^{2} \mu m_{i} \cdot\left(u_{i}-U\right)}{n Q^{2} Q_{i}^{2} e^{4} \ln \Lambda G_{1}(x)} .
$$

Here, $\ln \Lambda$ is again the Coulomb-logarithm (Spitzer 1956), and $G_{1}(x)$ denotes the function

$$
G_{1}(x)=\frac{\operatorname{erf}(x)-\operatorname{xerf}^{\prime}(x)}{2 x^{2}}
$$

in which erf $(x)$ is the error function and erf $f^{\prime}(x)$ is its derivative with respect to $x$. The argument $x$ is

$$
x=\frac{u_{i}-U}{\left(\frac{2 k T_{i}}{m_{i}}+\frac{2 k T}{m}\right)^{1 / 2}},
$$

and $\mu=: \frac{m m_{i}}{m+m_{i}}$. To illustrate the approach of a differentially streaming minor species to a distribution of field particles, consider the following simplified example: close to equilibrium the function $G_{1}(x)$ can be approximated with

$$
G_{1}(x \rightarrow 0) \approx \frac{2}{3 \sqrt{\pi}} \cdot x-\frac{2}{5 \sqrt{\pi}} \cdot x^{3},
$$

which leads to an expression for the speed relaxation time, which is relevant for a "hot" plasma, where thermal speeds are larger than the relative drifts between different species:

$$
t_{\mathrm{i}, \mathrm{eq}, \mathrm{speed} \rightarrow 0}=\frac{3 \pi^{3 / 2} \epsilon_{0}^{2} \mu m_{i}\left(\frac{2 k T_{i}}{m_{i}}+\frac{2 k T}{m}\right)^{3 / 2}}{n Q^{2} Q_{i}^{2} e^{4} \ln \Lambda \cdot\left(1-\frac{3}{5} \cdot \frac{\left(u_{i}-U\right)^{2}}{\left(\frac{2 k T_{i}}{m_{i}}+\frac{2 k T}{m}\right)}\right)}
$$

which is abbreviated to

$$
\tau_{\mathrm{eq}}(x) \approx \frac{A}{1-B x^{2}}
$$

Here, $x$ is again the difference of speeds in relation to the corresponding thermal velocities. From this it is possible to formulate a simple differential equation 
ruling the evolution of speed differences towards equilibrium, taking into account the steady decrease of equilibration times as given by (10) or (9)

$$
\frac{\partial f(x, t)}{\partial t}=\frac{f(x+\mathrm{d} x, t)}{\tau(x+\mathrm{d} x)}-\frac{f(x, t)}{\tau(x)},
$$

leading to

$$
\frac{\partial^{2} f(x, t)}{\partial t \partial x}=-\frac{2 B x f(x, t)}{A}+\frac{\left(1-B x^{2}\right)}{A} \frac{\partial f(x, t)}{\partial x} .
$$

The solution, $f(x, t)$, of Eq. (12) is illustrated in Fig. 4. Starting with an initially normally distributed histogram of observations $f(x, 0)$, the approach of the histogram $f(x, t)$ towards an equilibrium is shown. As time proceeds, the number of cases with small velocity differences increases dramatically on the account of cases with moderate velocity differences, whereas the number of cases with large velocity differences remains virtually unchanged.

We have already pointed out in the context of helium velocity distributions in Fig. 1 that velocity distributions of minor species in slow solar wind are shaped by Coulomb collisions. Another illustration for this observation

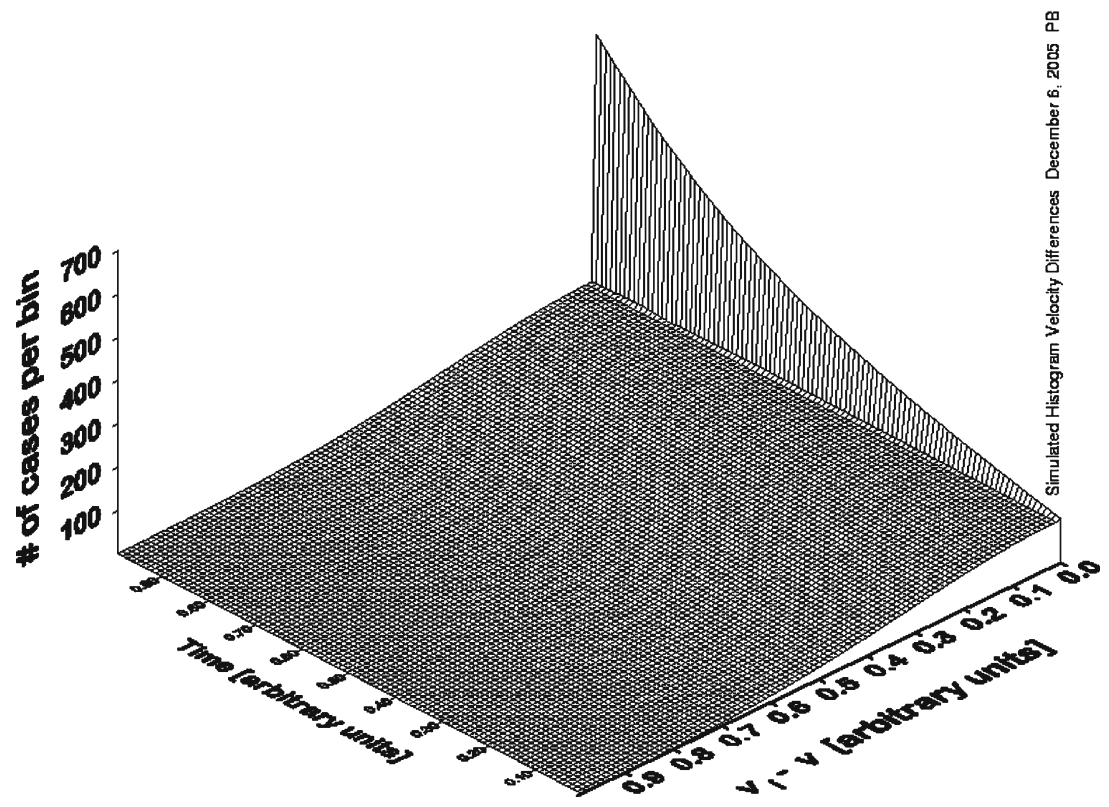

Fig. 4 Illustration of an example of a solution of Eq. (12): as time proceeds from right to left, an originally gaussian histogram of velocity differences evolves into a histogram, which becomes strongly peaked towards small velocity differences, whereas cases with large velocity differences flatten slowly towards a state reminiscent of panel 1 in Fig. 3 

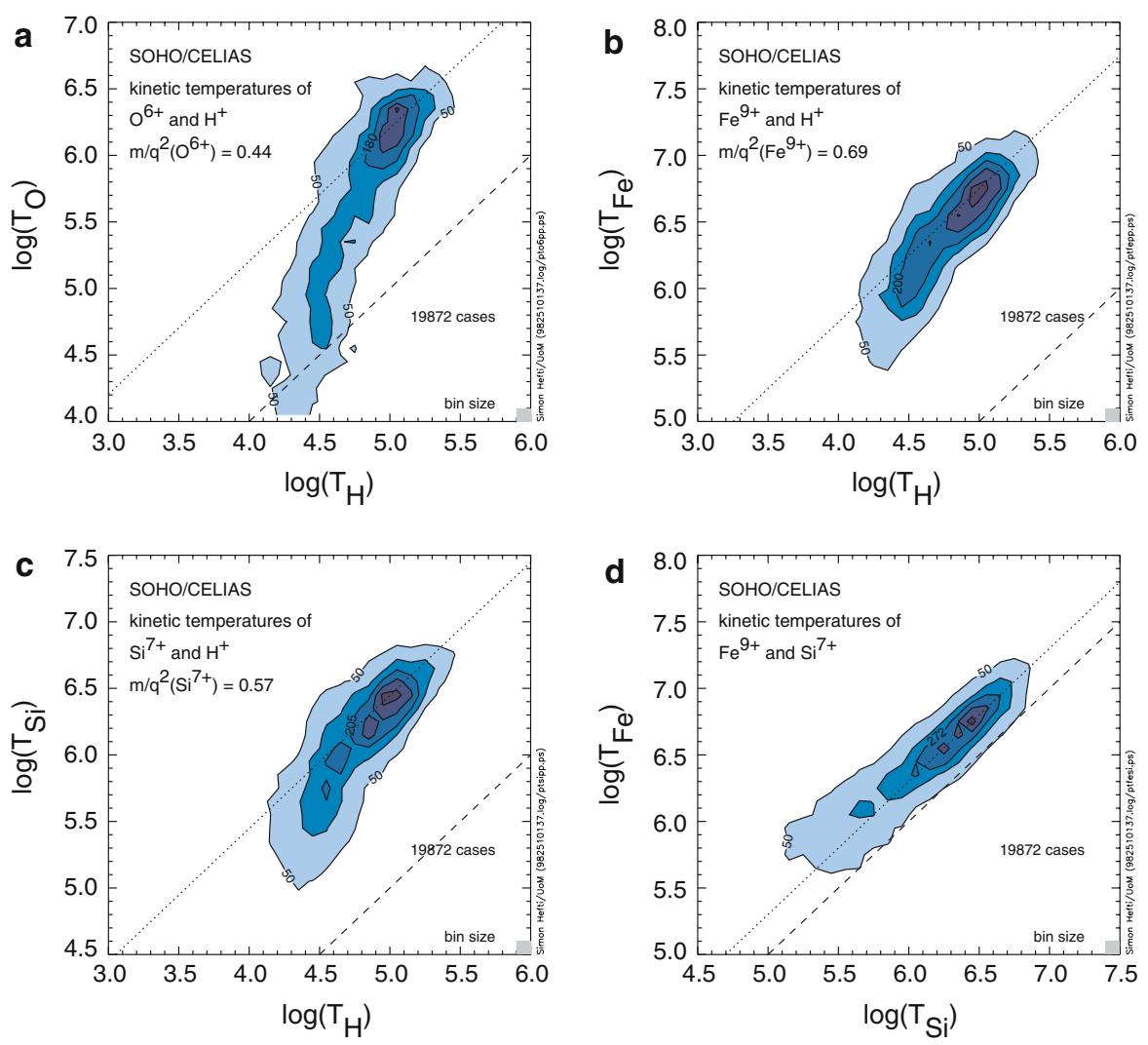

Fig. 5 Histograms of kinetic temperatures of minor ions observed with SOHO/CELIAS (from Hefti et al. (1998)). In high speed regimes, i.e., at elevated kinetic temperatures the temperatures are usually following the rule $T_{i} \propto m_{i}$, indicated by the dotted lines. In low-speed regimes and at low temperatures, temperatures appear more closely to the dashed line which indicates energy equipartition among minor species, probably as a consequence of Coulomb collisions. Note that species with the higher Coulomb drag factor (lower $m / q^{2}$ value) approach the equipartition line more closely at low temperatures (and low speeds)

has been provided by Hefti et al. (1998), from which we show some contour plots of histograms in Fig. 5. It is most clearly seen in the correlation of the kinetic temperatures of $\mathrm{O}^{6+}$ versus $\mathrm{H}^{+}$, where a significant fraction of observations indicate $T\left(\mathrm{O}^{6+}\right) \approx T\left(\mathrm{H}^{+}\right)$, typical of collision-dominated regimes. For all species the coronal-hole-associated regimes produce elevated kinetic temperatures with $T_{i} \propto m_{i}$, indicative for wave-particle interaction in the corona. This observation is somewhat counterintuitive as coronal-hole-associated solar wind is well known to be correlated with relatively low electron temperatures. As indicated at the end of Sect. 2, preferential heating of minor ions near the ion-cyclotron resonance, is considered to be the main agent responsible for this apparent paradoxon. 


\section{Observations of charge states}

The observation that elements in the solar corona appear in elevated charge states presented a difficult puzzle to astronomers in the first half of the twentieth century. Early on, the spectra of highly charged species such as Fe XII were not known and, furthermore, intuition was in conflict with the hypothesis that the tenuous solar corona would be so much hotter than the solar atmosphere. On the basis of first principles, one did not expect highly ionized species in the corona.

The interesting feature about charge states in the solar wind is their diagnostic potential at remote sites. Charge states of minor species are established in the inner corona by collisions with hot electrons. As an ion moves out of the corona, the ambient coronal electron density decreases, and collisions become so rare that the charge state of an ion no longer adapts itself to the ambient electron temperature by recombination, and consequently it "freezes". Hundhausen et al. (1968a), and later, Owocki et al. (1983) have elaborated on the details of charge-state abundances as diagnostic tools.

Setting up the balance of charge states of an element of atomic number $Z$ and assuming a steady-state outflow, one obtains a system of conservation equations for each charge state $i$

$$
\nabla \cdot\left(n_{i} v_{i}\right)=n_{\mathrm{e}}\left[n_{i-1} C_{i-1}-n_{i}\left(C_{i}+R_{i}\right)+n_{i+1} R_{i+1}\right], \quad i=0,1, \ldots Z,
$$

with $n_{i}$ and $v_{i}$ denoting density and flow to velocity of species $i$. $C_{i}$ and $R_{i}$ are the collisional ionization and the electron recombination rates, respectively, removing a particle into the next lower or next higher charge state. As is evident from (13), the set of $Z+1$ equations (including charge state 0 for neutrals) forms a system of equations, which conserves the flux of the element $Z$. The rates for Maxwellian electron velocity distributions $C_{i}$ and $R_{i}$ are found in the work of Arnaud and Rothenflug (1985), Arnaud and Raymond (1992) and in Voronov (1997). ${ }^{2}$

For the case of a weakly expanding plasma at high density, where a static equilibrium of charge states is maintained, one can infer so-called "freezing-in electron temperatures" $T_{\mathrm{e}}$, i.e., from couples of ionic abundances:

$$
\frac{n_{i}}{n_{i+1}}=\frac{R_{i+1}\left(T_{\mathrm{e}}\right)}{C_{i}\left(T_{\mathrm{e}}\right)}, \quad i=0,1, \ldots Z
$$

\subsection{Charge states as diagnostics for coronal conditions}

With a given ratio $n_{i} / n_{i+1}$ Eq. (14) can be resolved for $T_{\mathrm{e}}$. In the literature freezein temperatures, such as defined through Eq. (14), are often used as parameters

\footnotetext{
2 See also http://www.pa.uky.edu/ verner/fortran.html.
} 
for characterizing coronal conditions, rather than reporting abundance ratios of charge states; although in this case the condition of static equilibrium is hardly ever fulfilled. Different charge couples will give different temperatures, because the freezing conditions do not only depend on the coronal temperature but on the magnitudes of rates and - more precisely - also on the electron densities involved. For instance, large ions exhibit large cross-sections for electron capture and, hence, freeze at lower densities further out in the corona, where usually lower temperatures prevail. Comparing charge-state abundances from different species, it is possible to routinely infer coronal temperature gradients. Owocki et al. (1983) defined an electron freezing density equaling the ionization-recombination exchange time $\left(\tau_{i \leftrightarrow i+1} \equiv \frac{1}{n_{\mathrm{e}}\left(R_{i+1}+C_{i}\right)}\right)$ to the expansion time at the freezing distance

$$
n_{\mathrm{e}}\left(r_{f}\right)=n_{f}=\left[\frac{n_{E} v_{E}\left(A_{E} / A_{F}\right)}{H\left(R_{i+1}+C_{i}\right)}\right]^{1 / 2},
$$

where $n_{E} v_{E}$ is the ion flux at $1 \mathrm{AU}$ and $A_{E} / A_{F}$ is the ratio of the cross-section of the flow-tube at $1 \mathrm{AU}$ over its cross-section at the freezing distance.

An illustration of the "freezing" concept for charge states is given in Fig. 6, where the evolution of charge states of $\mathrm{Mg}$ from the solar surface to $3 \mathrm{R}_{\odot}$ heliocentric distance is shown. This so-called "dino-plot" uses the format of diagrams applied in palaeontology, where certain species expand on the account of others and will again diminish and become extinct after a certain period. Under conditions frequently found in low-speed solar wind, magnesium freezes typically to the helium-like $\mathrm{Mg}^{10+}$ because further ionization would require electrons at energies in excess of $1.7 \mathrm{keV} \approx 20 \mathrm{MK}$.

Owocki and Scudder (1983) investigated the influence of non-Maxwellian electron tails on the freezing-in properties of oxygen and iron and on possible biases when inferring electron temperatures under the assumption of a Maxwellian distribution. However, they did not determine quantitative limits on the importance of non-Maxwellian tails from observations. Dzif̌ćáková (1998, 2000) and Porquet et al. (2001) investigated ionization equilibria in coronal plasmas with non-Maxwellian velocity distributions. Ko et al. (1996), using Ulysses/SWICS data, were able to demonstrate that non-Maxwellian tails had no significant influence on the charge-state distributions, notably on those of oxygen and carbon, which are most sensitive due to the large ionization potentials of their K-shell electrons. Using data from the CELIAS experiment on SOHO, Aellig et al. (1998) could provide firm upper limits on the contribution of "hot" electrons ("hot" meaning $T_{\text {hot }}=10 T_{\mathrm{e}}$ ) to the ionization balance of oxygen and iron. These contributions amount at most to $5 \%$.

With the enhance efficiency and duty cycle of CELIAS/CTOF on the threeaxis- stabilized SOHO spacecraft, it was also possible to routinely determine coronal temperature gradients and to continuously measure coronal freezing-in densities with a time resolution of typically $25 \mathrm{~min}$. Aellig et al. (1997a) used 


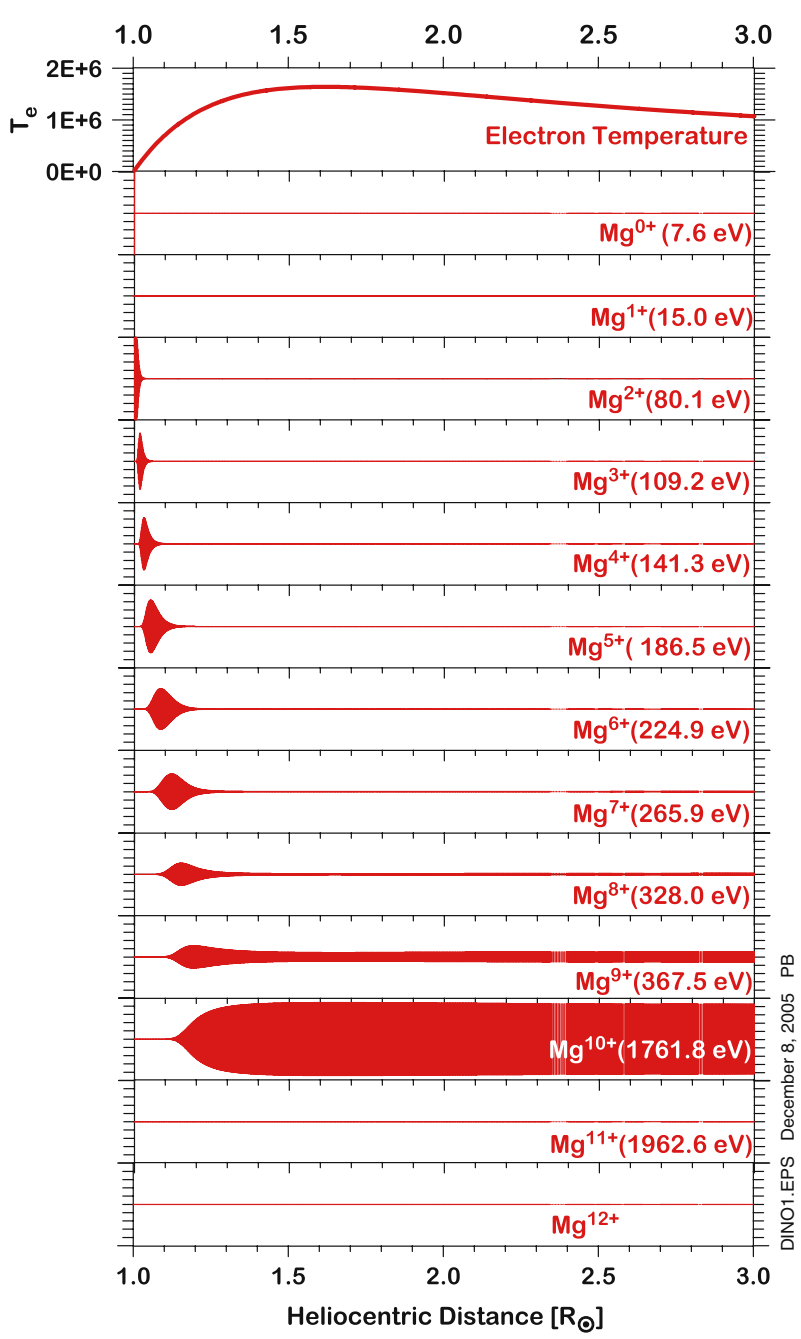

Fig. 6 Evolution of charge states of Mg. The topmost panel shows the electron temperatures in the underlying model, which describes a typical expansion of low-speed solar wind. The other panels illustrate the change from initially neutral $\mathrm{Mg}$ to $\mathrm{Mg}^{12+}$. The dominant charge state after freezing is $\mathrm{Mg}^{10+}$ a typical species found in the solar wind

a scheme parameterizing the radial electron-density dependence, temperature profile, and the speed dependence to fit-observed charge states. Two examples are shown in Fig. 7.

\subsection{Exotic charge-state distributions}

At several instances, substantial amounts of singly ionized helium have been found to coexist with typical solar wind species such as $\mathrm{O}^{6+}$ (Schwenn et al. 

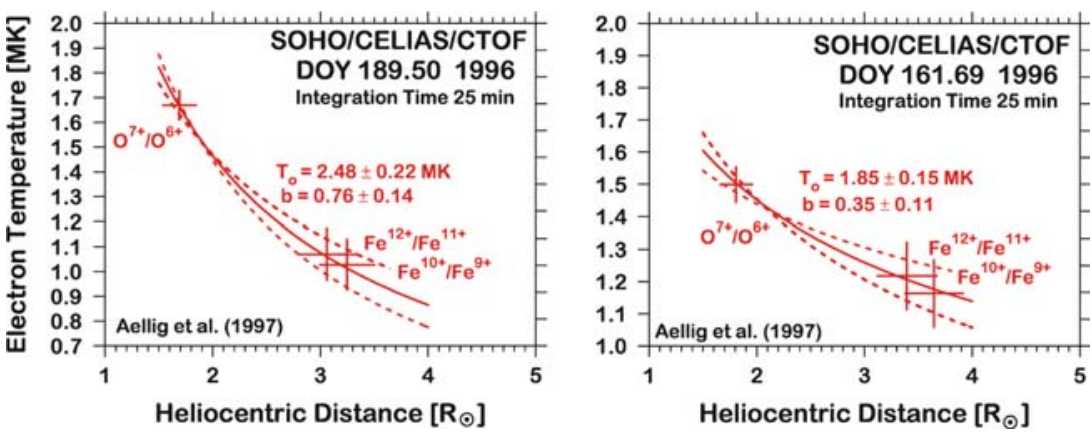

Fig. 7 Coronal temperature profile derived from observations of oxygen and iron charge states with SOHO/CELIAS (adapted from Aellig et al. (1997a)). Such profiles could be derived routinely with high time resolution. The left panel shows a case with a rather steep profile and elevated electron temperatures up to $1.7 \times 10^{6} \mathrm{~K}$. Such profiles are typical for low-speed solar wind. The right-hand side panel illustrates a flatter profile, representative for high-speed, coronal-hole-associated solar wind with a maximum near $1.5 \times 10^{6} \mathrm{~K}$

1980, Gosling et al. 1980). This cannot be explained in terms of a steady-state flow through a 1-2 MK hot corona. More recently, weakly ionized species have been detected flowing together with otherwise typical solar-wind charge states in combination with strong excesses of ${ }^{3} \mathrm{He}^{++}$(Ho 1998, Ho et al. 2000). Since these events of anomalous charge states were always observed in correlation with coronal mass ejections, Neukomm and Bochsler (1996) proposed rapid adiabatic expansion of magnetic plasmoid bubbles, associated with coronal mass ejections, to be the cause for the coexistence of weakly and strongly charged species.

\subsection{Charge states as tracers for heliospheric processes}

As an example for the potential of compositional features to serve as "hydrological" tracers to map solar-wind streams back to the Sun, we mention the work of Wimmer-Schweingruber et al. (1997), who succeeded with surgical precision to identify interfaces between high-speed streams and interstream flows by means of the $\mathrm{Mg} / \mathrm{O}$-abundance ratio and by means of the very sensitive change in the charge state of oxygen, which always appears to be associated with the elemental composition change. In a similar study, Aellig et al. (1997b) used SOHO/CELIAS-data with 5 min time resolution to constrain possible magnetic field-linebraiding in the interplanetary medium. These authors investigated correlated features of charge states of iron ions and found an upper limit along the path (along $z$ ) from the location of charge-state freezing in the inner corona to the site of observation at $1 \mathrm{AU}$. They found

$$
\left.\frac{\left\langle(\Delta x)^{2}\right\rangle}{\Delta z}\right|_{R=1 \mathrm{AU}} \leq 9 \times 10^{7} \mathrm{~m}^{2} / \mathrm{m} .
$$




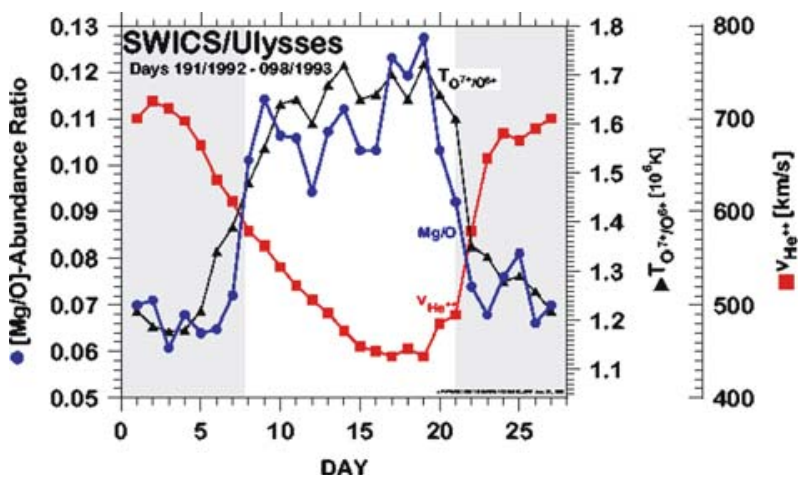

Fig. 8 Superposed epoch analysis of Ulysses/SWICS data covering ten solar rotations (adapted from Geiss et al. (1995)). The three measured parameters are established in three different domains of the solar wind: the $\mathrm{Mg} / \mathrm{O}$-abundance ratio (blue full circles) is fixed in the source region of the solar wind, i.e., in the chromosphere. The oxygen freezing temperature (black triangles) is established in the corona near the temperature maximum (see discussion above). The solar-wind velocity (red squares) is modified by wave-particle interaction and stream-stream interactions throughout the heliosphere. During the period of observation, Ulysses was moving at low heliographic latitudes cruising between the southern coronal hole and the streamer belt, which led to a repetitive high-speed wind stream (gray-shaded area in the plot) and the region near the repetitive current sheet (marked white). Note that the compositional features show rapid changes at the fringes while the kinetic property (solar-wind speed) changes smoothly

More recently, Schwadron et al. (2005) undertook a detailed investigation of compositional and dynamic properties of transitions from coronal-hole-associated solar wind to slow wind, such as illustrated in Fig. 8. Using a model, which involves the classical two sources of solar wind on the solar surface, coronal holes and the active streamer belt, they succeeded to reproduce all facets of solar-wind properties from the Sun to $5 \mathrm{AU}$. Their model considers foot-point motions of magnetic fields on the Sun, and with a newly developed concept of connecting B-fields, which carry slow wind and coronal-hole associated wind across the separating boundary layer, the two wind types are mixed over a limited range of heliomagnetic latitudes. This work presents another impressive case for the diagnostic power of compositional features.

\section{Elemental abundances}

\subsection{Observations}

Extensive literature exists on elemental and isotopic abundances in the corona and in the solar wind. Many of the compilations and reviews relate these abundances to photospheric composition. Originally, the most reliable results on coronal abundances have been obtained from solar energetic particles, i.e., from the so-called gradual events, which accelerate coronal particles with outgoing shocks generated by expanding coronal mass ejections. Summarizing all the evidence accumulated during the last decades comes close to an attempt 


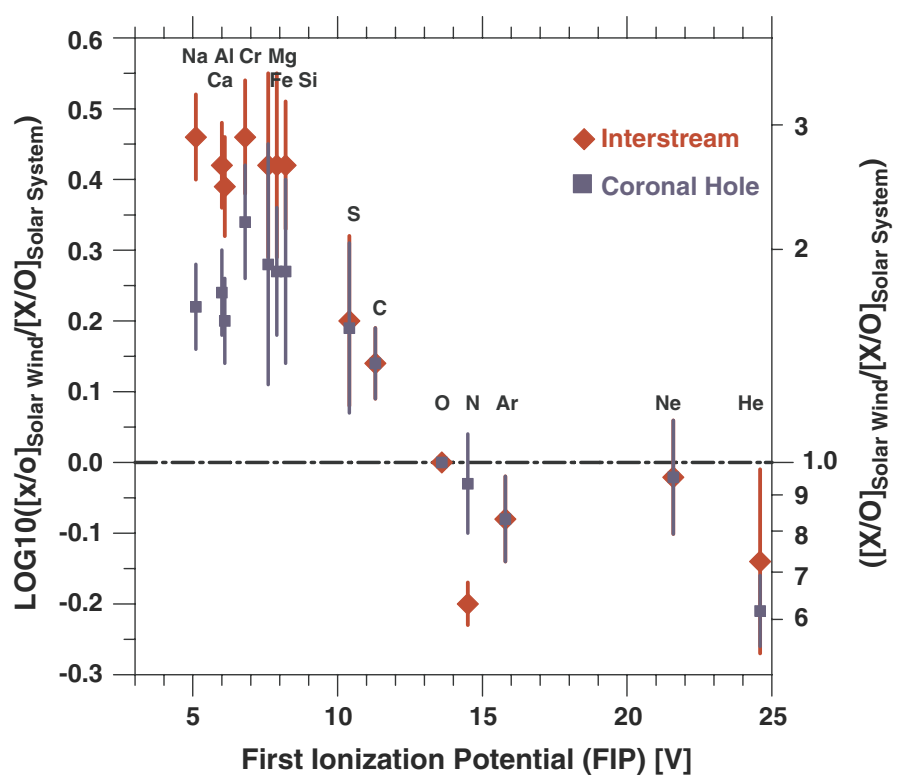

Fig. 9 Elemental enhancement factors of solar-wind abundances over typical solar system abundances for interstream- and coronal-hole-type flows versus first ionization potential

to hit a moving target; one reason being that the solar wind itself varies its composition, e.g., with the phase of the solar cycle.

The most prominent mechanism to modify solar photospheric abundances to coronal and solar-wind abundances is the so-called "FIP" effect illustrated in Fig. 9. Comparing solar wind abundances (as listed, e.g., in Table 2) and with solar photospheric or meteoritic abundances groups them according to first ionisation potentials (FIP's). In Fig. 9 enrichment factors have been calculated by dividing the elemental ratio of an element to oxygen in the solar wind (interstream-type or coronal-hole-associated wind) to the corresponding ratio in the solar system. In the slow-interstream solar wind the low-FIP elements show typical enhancements over solar-system abundances clustering around a factor of 2.5-3 - forming the so-called low-FIP plateau. The low-FIP plateau appears at a lower level for coronal-hole-associated solar wind. On the other hand, highFIP elements are usually depleted in the solar wind relative to oxygen. At least in the case of helium the amount of depletion is variable and possible reasons for this depletion will be discussed in the following.

The $\mathrm{He} / \mathrm{H}$-abundance ratio has been known for a long time to vary sometimes over orders of magnitudes - depending on the solar-wind regime. Depending on the period of observations one might obtain different results, just because one might look at different aliquots. Different instruments with different data reduction procedures have been involved in different studies at different phases of the solar activity cycle. Confidence in the fidelity of the instrument functions, the instrument calibration and the data reduction procedures applied to 
different experiments depends to a significant degree on the experience and the psychological constitution of the authors involved.

Studying the literature, one cannot avoid the conclusion that the systematic uncertainties of instrument functions are more often than not underestimated and falsely attributed to real variations in the solar wind. This becomes evident, when comparing the apparent variability of in situ data with the results obtained through laboratory measurements of the Apollo foils. ${ }^{3}$ Superposed to these difficulties - when relating coronal or solar-wind abundances to solar abundances - comes the fact that photospheric abundances are subject to complex model assumptions on radiative transfer and 3-D dynamics of optically unresolved photospheric and chromospheric structures. Again, more often than not, these complications have been underestimated, correspondingly, the uncertainties of photospheric abundances have often been underestimated (and in some cases even not stated). Also substantial revisions up to an order of magnitude have occurred, inflicting revisions also on fractionation factors derived from solar-wind and coronal abundances. We note in passing that model-invariant abundance ratios, e.g. relating high- and low-FIP elements, which compare abundances derived from fewer lines of different elements but originating under comparable photospheric conditions, would be more useful for this type of investigations than comprehensive lists of "solar elemental abundances".

Table 1 is a compilation of solar-wind abundances reported in the literature. All data are in reference to the abundance of oxygen. The uncertainties have been estimated from statistical fluctuations to the one-sigma level but do also include systematic uncertainties. The third column contains the so-called interstream (low-speed) solar-wind composition, predominantly flowing at the fringes of closed field regions (see the article by Schwadron et al. (2005) for an up-to-date description). The fourth column represents typical abundances for coronal-hole-associated (fast) solar wind. The last column is for reference: it lists the coronal abundances derived from solar energetic particle (SEP) abundances by Reames (1994). As outlined previously, these data have been inferred from so-called large gradual events, i.e., coronal material accelerated at shocks of coronal mass ejections. Because these particles arrive with much higher energies than solar-wind particles, the composition of such flows can be measured more precisely than the solar-wind composition, although strong but rather systematic $\mathrm{Q} / \mathrm{M}$-fractionation - variable from event to event - occurs.

There exists ample literature also on coronal mass ejection (CME) abundances, which contribute a non-vanishing fraction to the equatorial solar-wind flow. Judging from the compilation of Webb and Howard (1994), one arrives at the conclusion that taken over a full solar activity cycle, typically $10 \%$ of the in-ecliptic solar-wind mass flux is related to coronal mass ejections. Recently,

\footnotetext{
3 Even though the Apollo foil exposures lasted from $77 \mathrm{~min}$ to $45 \mathrm{~h}$, and consequently, must have smeared some of the short-term variations of the solar-wind composition, the variability reported in many in situ observations cannot be reconciled with the considerably smaller variability observed with the Apollo foils (Bochsler and von Steiger 2004).
} 
Table 1 Solar-wind elemental abundances

\begin{tabular}{|c|c|c|c|c|}
\hline Element & Interstream & Coronal hole & References & $\begin{array}{l}\text { SEP corona } \\
\text { (Reames 1994) }\end{array}$ \\
\hline $\mathrm{He}$ & $90 \pm 30$ & $75 \pm 10$ & $\begin{array}{l}\text { Bochsler (1984) } \\
\text { Bochsler et al. (1986) } \\
\text { von Steiger et al. (2000) }\end{array}$ & $57 \pm 3$ \\
\hline $\mathrm{C}$ & $0.68 \pm 0.07$ & $0.68 \pm 0.07$ & von Steiger et al. (2000) & $0.465 \pm 0.009$ \\
\hline $\mathrm{N}$ & $0.078 \pm 0.005$ & $0.114 \pm 0.021$ & $\begin{array}{l}\text { Gloeckler et al. (1986) } \\
\text { von Steiger et al. (2000) }\end{array}$ & $0.124 \pm 0.003$ \\
\hline $\mathrm{O}$ & $\equiv 1$ & $\equiv 1$ & & $\equiv 1$ \\
\hline $\mathrm{Ne}$ & \multicolumn{2}{|c|}{$0.14 \pm 0.03$} & $\begin{array}{l}\text { Geiss et al. (1972) } \\
\text { Bochsler et al. (1986) } \\
\text { Collier et al. (1996) }\end{array}$ & $0.152 \pm 0.004$ \\
\hline $\mathrm{Na}$ & $0.0090 \pm 0.0015$ & $0.0051 \pm 0.0014$ & Ipavich et al. (1999) & $0.0104 \pm 0.0011$ \\
\hline $\mathrm{Mg}$ & $0.147 \pm 0.050$ & $0.106 \pm 0.050$ & von Steiger et al. (2000) & $0.196 \pm 0.004$ \\
\hline $\mathrm{Al}$ & $0.0119 \pm 0.003$ & $0.0081 \pm 0.0004$ & Bochsler et al. (2000) & $0.0157 \pm 0.0016$ \\
\hline $\mathrm{Si}$ & $0.140 \pm 0.050$ & $0.101 \pm 0.040$ & $\begin{array}{l}\text { Bochsler (1989) } \\
\text { Galvin et al. (1992) } \\
\text { von Steiger et al. (2000) }\end{array}$ & $0.0152 \pm 0.0004$ \\
\hline $\mathrm{S}$ & \multicolumn{2}{|c|}{$0.050 \pm 0.015$} & von Steiger et al. (2000) & $0.0318 \pm 0.007$ \\
\hline $\mathrm{Ar}$ & $0.0031 \pm 0.0008$ & $0.0031 \pm 0.0004$ & $\begin{array}{l}\text { Cerutti (1974) } \\
\text { Weygand et al. (2001b) } \\
\text { Geiss et al. (2004) }\end{array}$ & $0.0033 \pm 0.0002$ \\
\hline $\mathrm{Ca}$ & $0.0081 \pm 0.0015$ & $0.0053 \pm 0.0010$ & $\begin{array}{l}\text { Kern et al. (1997) } \\
\text { Kern (1999) } \\
\text { Wurz et al. (2003) }\end{array}$ & $0.0106 \pm 0.0004$ \\
\hline $\mathrm{Cr}$ & $0.0020 \pm 0.0003$ & $0.0015 \pm 0.0003$ & Paquette et al. (2001) & $0.0021 \pm 0.0003$ \\
\hline $\mathrm{Fe}$ & $0.122 \pm 0.050$ & $0.088 \pm 0.050$ & $\begin{array}{l}\text { Schmid et al. (1988) } \\
\text { Aellig et al. (1999a) } \\
\text { Aellig et al. (1999b) } \\
\text { von Steiger et al. (2000) }\end{array}$ & $0.134 \pm 0.004$ \\
\hline
\end{tabular}

Richardson and Cane (2004) undertook an extensive study to identify CME's on the basis of various compositional features. A similar study was performed by Reisenfeld et al. (2003) to establish an algorithm preventing CME's from "contaminating" samples of coronal-hole-associated wind on the Genesis spacecraft. Both studies use solar-wind speed as an ordering criterion. From the Reisenfeld study one realizes that low-speed CME's are most difficult to be distinguished from the ambient low-speed solar wind, whereas at high speeds the most clearcut identification is possible with elevated charge states of oxgyen and elevated $\mathrm{Mg} / \mathrm{O}$-abundance ratios. The composition of this type of flows varies substantially from event to event and, because such events exhibit complicated spatial structures, any reported measurement depends heavily on the location of the relevant in situ instrument relative to the passage of the ejecta.

In many aspects, CME-related flow reflects most closely low-speed solar wind, except for the general enhancement of $\mathrm{He}$, typically $[\mathrm{He}] /[\mathrm{H}] \geq 6 \%$. One wonders, whether CME-related solar wind needs to be considered as an independent type of flow, or whether low-speed solar wind reflects a composition, produced by a multitude of small-scale CME's, which dissolve in the inner 
corona, while only the large-scale CME's survive out to spacecraft observations and remain, hence, distinguishable as independent events. A detailed discussion and a solid investigation of these relationships on statistical grounds is beyond the scope of this article.

The purpose of Table 1 is to give a best estimate of solar-wind abundances. It is biased on the author's prejudices and notions on the FIP-process. It is not intended to make an attempt to derive a FIP-fractionation factor or to test the flatness of a FIP plateau. Multiple entries in the reference column of Table 1 indicate that many independent references have been considered for this compilation. Many abundance parameters reported in the literature have been derived with different purposes in mind. We have used the abundances of the major refractory elements, $\mathrm{Mg}+\mathrm{Si}+\mathrm{Fe}$, given in Table 1 of the article of von Steiger et al. (2000) to derive lumped low-FIP fractionation factors. The data provided by these authors have the virtue that they have all been measured with one singular experiment from the same mission and have been evaluated by one team using the same instrument function. This can, of course, not completely exclude some systematic (instrumental) biases. Table 1 contains also some entries on more recent determinations of elemental abundances. Bochsler et al. (2000) provided a measurement for the $\mathrm{Al} / \mathrm{Mg}$-abundance ratio and quoted $\mathrm{Na} / \mathrm{Mg}$ abundances given by Ipavich et al. (1999). Similarly, the value for chromium of Paquette et al. (2001) was related to the iron abundance. For neon and argon the most reliable values date back to the measurements with the Apollo foils of Geiss et al. (1972). These abundances - all from high-FIP elements - which apparently do not undergo severe FIP fractionation have been referred to the coronal-hole-associated solar-wind helium abundance. Finally, we have related the $\mathrm{Ca}$ abundance to its mass neighbor Ar, heavily relying on the Ar/Ca ratio derived by Weygand et al. (2001b). Note, that our triangulation through low-FIP elemental abundances has not included $\mathrm{Ca}$. Hence, the Ca abundance provides an independent test on the level of the previously defined low-FIP plateau with the abundances of oxygen and argon.

Table 2 presents the solar-wind abundances in the logarithmic dex scale with the oxygen abundance fixed for all columns at 8.83. The solar-system values have been adopted from the work of Grevesse and Sauval (1998). The fifth and the seventh column give the deviation of the solar-wind abundance from the solar abundance (also in a logarithmic scale) for interstream and for coronal-hole-associated solar wind, respectively. These deviations group now in the relatively narrow range from 0.39 to 0.46 (corresponding to fractionation factors of 2.5-2.9) for interstream wind and low-FIP elements such as $\mathrm{Na}, \mathrm{Mg}$, $\mathrm{Si}, \mathrm{Al}, \mathrm{Fe}$, etc. This good match is, of course, somewhat artificial due to our lumping procedure for the major elements $\mathrm{Mg}, \mathrm{Si}$, and $\mathrm{Fe}$, described in the previous paragraph. However, note that the $\mathrm{Ca}$ abundance is fully independent of this procedure and gives an excellent match, as the other high-FIP elements $\mathrm{Na}, \mathrm{Al}$, and $\mathrm{Cr}$ confirm the absolute flatness of the high-FIP plateau. The latter three elements having been determined independently and linked to $\mathrm{Mg}$ and $\mathrm{Fe}$, respectively. High-FIP and intermediate elements (He, N, Ne, etc.) cluster around 0 . Unfortunately there is no independent solar-system reference for Ar. 
Table 2 Solar-wind elemental abundances and solar-system abundances (for references of solarwind measurements, see Table 1)

\begin{tabular}{lrlcccc}
\hline Element & $\begin{array}{r}\text { FIP } \\
{[\mathrm{V}]}\end{array}$ & $\begin{array}{l}\text { Solar system } \\
(\text { Grevesse and Sauval 1998) }\end{array}$ & Interstream & IS/SS & Coronal hole & CH/SS \\
\hline $\mathrm{He}$ & 24.6 & {$[10.92]^{\mathrm{a}, \mathrm{b}}$} & $10.78 \pm 0.13$ & -0.14 & $10.71 \pm 0.05$ & -0.21 \\
$\mathrm{C}$ & 11.3 & 8.52 & $8.66 \pm 0.05$ & +0.14 & $8.66 \pm 0.05$ & +0.14 \\
$\mathrm{~N}$ & 14.5 & 7.92 & $7.72 \pm 0.03$ & -0.20 & $7.89 \pm 0.07$ & -0.03 \\
$\mathrm{O}$ & 13.6 & $\equiv 8.83$ & $\equiv 8.83$ & $\equiv 0$ & $\equiv 8.83$ & $\equiv 0$ \\
$\mathrm{Ne}$ & 21.6 & $8.001^{\mathrm{c}}$ & $7.98 \pm 0.08$ & -0.02 & $7.98 \pm 0.08$ & -0.02 \\
$\mathrm{Na}$ & 5.1 & 6.32 & $6.78 \pm 0.06$ & +0.46 & $6.54 \pm 0.06$ & +0.22 \\
$\mathrm{Mg}$ & 7.6 & 7.58 & $8.00 \pm 0.13$ & +0.42 & $7.86 \pm 0.17$ & +0.28 \\
$\mathrm{Al}$ & 6.0 & 6.49 & $6.91 \pm 0.06$ & +0.42 & $6.73 \pm 0.06$ & +0.24 \\
$\mathrm{Si}$ & 8.2 & 7.56 & $7.98 \pm 0.09$ & +0.42 & $7.83 \pm 0.13$ & +0.24 \\
$\mathrm{~S}$ & 10.4 & 7.33 & $7.53 \pm 0.12$ & +0.20 & $7.52 \pm 0.12$ & +0.19 \\
$\mathrm{Ar}$ & 15.8 & {$[6.40]^{\mathrm{a}}$} & $6.32 \pm 0.06$ & & $6.32 \pm 0.06$ & \\
$\mathrm{Ca}$ & 6.1 & 6.35 & $6.74 \pm 0.07$ & +0.39 & $6.55 \pm 0.06$ & +0.20 \\
$\mathrm{Cr}$ & 6.8 & 5.67 & $6.13 \pm 0.08$ & +0.46 & $6.01 \pm 0.08$ & +0.34 \\
$\mathrm{Fe}$ & 7.9 & 7.50 & $7.92 \pm 0.13$ & +0.42 & $7.77 \pm 0.09$ & +0.27 \\
\hline
\end{tabular}

a Values in brackets no photospheric measurement exists

b We use the value for the outer convective zone of the Sun from helioseismological observations given by Däppen (1998) as reference

c Here, we adopt the Ne abundance derived by Holweger (2001). See Sect. 6.2 for an independent determination

The case of coronal-hole-associated FIP enrichments is less clear, however. According to Table 2 the enrichment factors for low-FIP elements group in the range from 1.6 to 2.2. The higher end is determined by the somewhat elevated Fe abundance (including $\mathrm{Cr}$, which has been tied to $\mathrm{Fe}$ ). Had we used the solar $\mathrm{Fe}$ abundance of Holweger (2001), the picture would be somewhat more consistent.

For reasons of internal consistency, we have exclusively used the photospheric or meteoritic values of Grevesse and Sauval (1998) in Table 2. In many cases meteoritic and photospheric values agree within less than $15 \%$. In cases of doubt and for non-volatile elements we have adopted the meteoritic value to be the "solar-system" value, implying that carbonaceous chondrites are faithful representatives of solar system matter, except for the noble gases and nitrogen.

Holweger (2001) and coworkers have carefully evaluated some key abundances, partly using 3-D hydrodynamical models of the solar atmosphere. Whereas there is generally good agreement for the low-FIP elements, which are less controversial anyway, because they can be inferred from meteoritic values, the revision of Holweger (2001) brings different values for the high-FIP elements, which are purely photospheric. The differences range up to 30\% (in the case of nitrogen). The computed fractionation factors IS/SS and CH/SS in Table 2 would converge somewhat better towards 0 , had we used Holwegers photospheric abundances for high-FIP elements except for the case of nitrogen. Note again, that the flatness of the high-FIP plateau in this table might be somewhat misleading, because some of the low-FIP abundances $(\mathrm{Mg}, \mathrm{Si}$, 
and $\mathrm{Fe}$ ) have been tied together using similar fractionation factors for all three elements. On the other hand $\mathrm{Na}, \mathrm{Al}$, and $\mathrm{Cr}$ have been tied to abundances of the major low-FIP elements, since refractory elements provide generally more reliable solar system references. The abundances of these minor elements confirm the flatness of the low-FIP plateau, but they do not provide independent proof for the validity of the FIP-enhancement factor in this table. Similarly, Weygand et al. (2001b) tied their Ar values to the solar-system Ca abundance. While von Steiger et al. (2000) clearly state that both solar-wind types, slow and fast, show a definite enrichment of low-FIP elements, Aellig et al. (1999b) take a cautious view and emphasize the difficulties relating solar-wind and coronal abundances to solid and reliable photospheric abundance ratios between low- and high-FIP elements. These doubts seem justified in view of the fact, that determinations of photospheric abundances rest heavily on model assumptions about the solar atmosphere with the corresponding uncertainties. Some of these uncertainties can be eliminated if photospheric abundance ratios rather than plain abundances are considered, as has been shown by Aellig et al. (1999b). Special difficulties in studying FIP-related fractionation are encountered with high-FIP elements, because, either there are no independent photospheric observations, or the solar-abundance values are based on coronal or solar-wind abundances. Another cautionary remark about such compilations, as the ones given here, is also in order: these values are statistical averages, and, considering the complicated mechanisms, which might be involved in the solar-wind feeding process, it would be surprising if a given regime would always reproduce exactly the same fractionation factor in the solar wind. This point has been strongly emphasized especially for the case of low-speed solar wind by the Ulysses/SWICS-Team ( von Steiger et al. 2000, Schwadron et al. 2005). A critical assessment of the variability of isotopic abundances follows in Sect. 6 .

\subsection{Modeling elemental fractionation}

The ordering of enrichment factors according to FIP puts the location of the elemental fractionation into the upper solar atmosphere, where elements are ionized and can be transferred into the transition region and into the corona. There is apparently a general consensus among the many different attempts for theoretical explanations on this point, but opinions on details of the processes disagree. This is no surprise in view of the complexity of the mechanisms involved. While von Steiger and Geiss (1989) elaborate on FIP separation in different magnetic loops using the action of gravity and acceleration to separate ions from neutrals, Schwadron et al. (1999) describe the solar-wind feeding process as a stochastic superposition of heating and separation of ions from neutrals in loops of different sizes. On the other hand, Marsch et al. (1995) invoke a more simple steady-state diffusion model, which separates ions from neutrals and feeds them into the solar wind in a two-step process. In the first step particles are ionized and, depending on the ionization rate, they are fed 
more or less efficiently to the second step, where ions are driven up into the solar-wind flow with another efficiency factor, which depends on Coulomb-friction with protons. The model of Schwadron et al. (1999) does not investigate the ionization process in detail but assumes charge-state equilibria of different species following collisional ionization and recombination at different levels in the transition region, as given by Arnaud and Rothenflug (1985). On the other hand, the models of Geiss and Bochsler (1985), von Steiger and Geiss (1989), and Marsch et al. (1995) use photoionization rates considering balances of different levels of atoms.

We have emphasized above that what is ultimately observed will be a statistical average, since a given solar-wind flux-tube might well be fed intermittently from different fractionation sites and all theories discussed so far rest on unrealistic assumptions and use too many free parameters. A more realistic approach to discuss elemental fractionation would involve dynamic ionization models as the ones developed by Carlsson and Stein $(1999,2002)$. And a more realistic model should reduce the number of free parameters and use detailed optical information on the dynamical state of the chromosphere instead. Such a model should consider time-dependent photoionization and collisional ionization in shocked environments. Needless to say that a detailed analysis involving elements with different ionization properties in a 3-D model requires a much more elaborate treatment than all models discussed so far.

The most important case of elemental fractionation between solar wind and the $\mathrm{OCZ}$ of the Sun, i.e., the case of the $\mathrm{He} / \mathrm{H}$ fractionation, is probably also the most difficult one. Helium with the abundant isotope, ${ }^{4} \mathrm{He}$, has two shortcomings with respect to discrimination against hydrogen: it has the highest FIP of all elements and its most abundant charge state in the solar wind, ${ }^{4} \mathrm{He}^{2+}$, has the least favorable Coulomb drag factor among the commonly observed species in the solar wind. We will discuss this in more detail in the context of isotopic fractionation.

\section{Isotopic abundances}

The most reliable information on the isotopic composition of the solar OCZ originates from solar-wind measurements. Optical observations can yield clues on the isotopic composition of the photosphere for a few elements, which can form molecules at photospheric conditions. Valuable isotopic composition data are also available from solar energetic particles (e.g., Cohen et al. 2000, Leske et al. 2001), however, SEPs show a relatively large variability in isotopic composition from event to event due to synchronously operating elemental and mass-dependent fractionation processes at different sites in the solar atmosphere. The most prominent evidence is the sometimes dramatic enhancement of the ${ }^{3} \mathrm{He} /{ }^{4} \mathrm{He}$ abundance ratio in impulsive flares, due to resonant heating of ${ }^{3} \mathrm{He}$ in the flare acceleration process. Cases with strong ${ }^{3} \mathrm{He}$ enrichments have occasionally also been found in solar-wind flows associated with coronal mass ejections (Ho et al. 2000). Possibly, such flows originate in magnetic 
flux-tubes, which were contaminated with resonantly heated plasma from nearby flare sites, as is evidenced also by the presence of anomalous charge states. In view of the many uncertainties related to the fractionation of SEPs and the large uncertainties of isotopic abundances from solar molecules, we will use exclusively meteoritic (and terrestrial) references for the following discussion.

\subsection{Observations}

Table 3 is a compilation of published data on the isotopic composition in the solar wind at the time of the submission of this review early in 2006. The solar-system values are mostly meteoritic. In the case of non-volatile elements very little variation between meteoritic, terrestrial, and lunar samples is observed. The corresponding experimental uncertainties (not given in Table 3) are tiny compared to solar-wind uncertainties. The digits given in Table 3 for solar-system ratios are significant. The uncertainties of the solar-wind values usually cover 1 standard deviation. We expect that the information pertinent to the solar wind might be outdated in several respects after publication of the complete Genesis mission results. However, applications and general conclusions will most likely remain the same.

For the solar system isotopic abundance ratio, ${ }^{14} \mathrm{~N} /{ }^{15} \mathrm{~N}$, we have used the recently published value of Abbas et al. (2004), confirming an earlier measure-

Table 3 Solar-wind isotope-abundances

\begin{tabular}{|c|c|c|c|}
\hline Isotope ratio & Solar wind & References & Solar system \\
\hline${ }^{4} \mathrm{He}:{ }^{3} \mathrm{He}$ & $\begin{array}{l}2,350 \pm 120 \\
2,450 \pm 460\end{array}$ & $\begin{array}{l}\text { Geiss et al. (1972) } \\
\text { Geiss et al. (2004) } \\
\text { Ogilvie et al. (1980b) } \\
\text { Coplan et al. (1984) } \\
\text { Bodmer and Bochsler (1998) } \\
\text { Gloeckler and Geiss (1998) }\end{array}$ & Not available \\
\hline${ }^{14} \mathrm{~N}:{ }^{15} \mathrm{~N}$ & $200 \pm 55$ & Kallenbach et al. (1998a) & $448 \pm 62$ \\
\hline${ }^{16} \mathrm{O}:{ }^{18} \mathrm{O}$ & $446 \pm 90$ & $\begin{array}{l}\text { Collier et al. (1998) } \\
\text { Wimmer-Schweingruber et al. (2001) }\end{array}$ & 498.8 \\
\hline${ }^{20} \mathrm{Ne}:{ }^{22} \mathrm{Ne}$ & $13.7 \pm 0.3$ & $\begin{array}{l}\text { Geiss et al. (1972) } \\
\text { Geiss et al. (2004) }\end{array}$ & Not available \\
\hline${ }^{20} \mathrm{Ne}:{ }^{22} \mathrm{Ne}$ & $13.8 \pm 0.7$ & Kallenbach et al. (1997) & Not available \\
\hline${ }^{20} \mathrm{Ne}:{ }^{21} \mathrm{Ne}$ & $411 \pm 40$ & $\begin{array}{l}\text { Geiss et al. (1972) } \\
\text { Geiss et al. (2004) }\end{array}$ & Not available \\
\hline${ }^{24} \mathrm{Mg}:{ }^{25} \mathrm{Mg}:{ }^{26} \mathrm{Mg}$ & $78.5 \pm 0.8: 10.2 \pm 0.5: 11.3 \pm 0.7$ & $\begin{array}{l}\text { Bochsler et al. (1996) } \\
\text { Kucharek et al. (1998) }\end{array}$ & 78.99:10.00:11.01 \\
\hline $\begin{array}{l}{ }^{28} \mathrm{Si}:{ }^{29} \mathrm{Si} \cdot{ }^{30} \mathrm{Si} \\
{ }^{36} \mathrm{Ar}:^{38} \mathrm{Ar}\end{array}$ & $\begin{array}{l}92.20 \pm 0.26: 4.54 \pm 0.20: 3.26 \pm 0.21 \\
5.3 \pm 0.3 \\
5.5 \pm 0.5\end{array}$ & $\begin{array}{l}\text { Wimmer-Schweingruber et al. (1998) } \\
\text { Cerutti (1974) } \\
\text { Geiss et al. (2004) } \\
\text { Weygand et al. (2001a) } \\
\text { Weygand et al. (2001b) }\end{array}$ & $\begin{array}{l}92.23: 4.67: 3.10 \\
5.32\end{array}$ \\
\hline $\begin{array}{l}{ }^{40} \mathrm{Ca}:{ }^{42} \mathrm{Ca}:{ }^{44} \mathrm{Ca} \\
{ }^{54} \mathrm{Fe}:{ }^{56} \mathrm{Fe}:{ }^{57} \mathrm{Fe} \\
{ }^{58} \mathrm{Ni}:{ }^{60} \mathrm{Ni}\end{array}$ & $\begin{array}{l}96.9 \pm 0.34: 0.76: 1.99 \pm 0.34 \\
6.20 \pm 0.34: 91.24 \pm 0.53: 2.28 \pm 0.46 \\
68.1: 25.9: 3.9\end{array}$ & $\begin{array}{l}\text { Kallenbach et al. (1998b) } \\
\text { Ipavich et al. (2001) } \\
\text { Ipavich (2006) }\end{array}$ & $\begin{array}{l}96.941: 0.647: 2.086 \\
5.8: 91.72: 2.2 \\
68.27: 26.10: 3.59\end{array}$ \\
\hline
\end{tabular}


ment of Owen et al. (2001) for the Jovian atmosphere. The solar-wind value differs substantially from Jupiter, and the two values are inconsistent with each other. It seems unlikely that the solar-wind value is so low due to isotopic fractionation; on the other hand, given the abundance of nitrogen in Jupiter's atmosphere it seems also unlikely that Jupiter differs by a large factor from the protosolar value, e.g., due to admixture of light nitrogen from grains and ices. Clarification might be obtained by re-evaluating the SOHO/CELIAS results, which now cover a full solar cycle.

In Table 3 we have not included measurements of iron isotopic abundances by Oetliker et al. (1997). Their results obtained with WIND/MASS differ significantly from solar system values and have not been confirmed by Ipavich et al. (2001) for the solar wind.

To unambiguously identify possible isotopic fractionation effects, the three abundant isotopes of magnesium have been used. Magnesium is a moderately refractory element, for which the solar elemental abundance agrees very well with the one for meteorites. Thus, it is safe to assume that no loss concomitant with isotopic fractionation has occurred in the relevant meteoritic samples. Furthermore, all solar system samples, including lunar and terrestrial ones, as well as meteorites attributed to Martian origin, show very similar isotopic magnesium compositions except for a tiny contribution from long-lived $\left(\tau_{1 / 2}=740,000\right.$ years $)$ radioactive ${ }^{26} \mathrm{Al}$, which decayed after solidification of the samples. Normal solar system matter, including the Sun, contain some minute amounts of fossil ${ }^{26} \mathrm{Al}$. Only a few calcium- and aluminum-rich early condensates in meteorites show some variability in ${ }^{26} \mathrm{Mg}$, indicating that ${ }^{26} \mathrm{Al}$ was still alive when the sample solidified (Lee et al. 1976).

Figure 10 is an illustration of derived solar-wind fractionation effects for a variety of moderately refractory elements. It appears from the compilation of Table 3 that no evidence for isotopic fractionation (to the limit of a few percent per mass unit) can be derived. However, this conclusion does not include all the available evidence. In situ experiments have the advantage that one can obtain considerably more insight than just computing long-time averages of parameters with the goal to compare them with meteoritic and photospheric abundances. It is possible to investigate the variability of fractionation parameters under different solar-wind regimes. If a systematic fractionation of isotopes really occurs in the solar wind, all theoretical evidence and the observations of the $\mathrm{He} / \mathrm{H}$ variability suggest, that there should be a trend in strength of this fractionation with other solar-wind parameters, e.g., with the solar-wind speed, the solar-wind proton flux or, most importantly, with the $\mathrm{He} / \mathrm{H}$-abundance ratio. Most theoretical models of the FIP fractionation lead to the conclusion that the FIP effect, basically being an atomic effect, will not fractionate isotopes in any significant manner. Hence, one would not expect to observe isotopic fractionation related to FIP enrichments or depletions. This leaves inefficient Coulomb drag, or mass-discriminating wave-particle interaction in the corona as prime candidate processes yielding such effects.

Studies specially designed to locate such effects have been carried out earlier. We refer to the work of the CELIAS-Team (Kallenbach et al. 1998c, Kucharek 


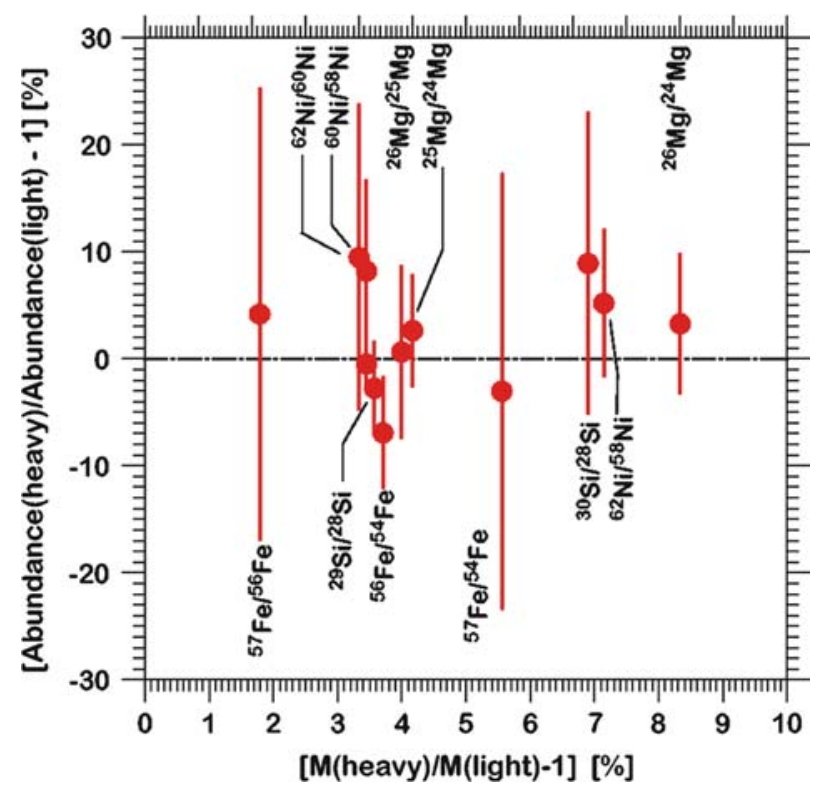

Fig. 10 Illustration of the compilation in Table 3. All refractory elements except Ca are included. Using unfractionated isotopic abundances of refractory elements in the solar system as baseline, a systematic isotopic fractionation effect in the solar wind is expected to reveal a systematic trend in this plot. No such fractionation has emerged at the level of accuracy obtained from in situ measurements

et al. 1998), who investigated possible trends of isotopic fractionation with the solar-wind speed. The observed trends point in the sense of heavier species being depleted in low-speed solar wind, just as one would expect from inefficient Coulomb drag. The measurements published so far, however, are still consistent with no trend at all. One encounters the same general picture, when comparing isotopic ratios from the foil experiments carried out during the Apollo missions Geiss et al. (1972) Wiens et al. (2004).

\subsection{Interpretation}

In order to make inferences on the isotopic composition of the OCZ of the Sun, one has to understand possible fractionation processes in the solar wind. As mentioned in the case of the solar-wind $\mathrm{He} / \mathrm{H}$-abundance ratio, such processes can produce substantial modifications - at least with respect to elemental abundances. The observed $\mathrm{He} / \mathrm{H}$ variability, or at least part of it, is generally attributed to inefficient Coulomb drag, and hence, as pointed out before, one would expect isotopic fractionation to correlate with the $\mathrm{He} / \mathrm{H}$ variability. The difficulty of allocating a clear cause to this effect lies in the fact, that ${ }^{4} \mathrm{He}^{++}$is not only the species with the least efficient Coulomb drag factor, but helium as an element, might be specially discriminated because of its exceedingly high FIP (and correspondingly elevated atomic excitation levels). 
Two approaches to distinguish the influence of the FIP effect from the influence of inefficient Coulomb drag can be envisioned. An investigation following the first approach is currently in preparation: it consists in the search for a correlation between the ionizing EUV-radiation flux in the source region and the $\mathrm{He} / \mathrm{H}$-abundance ratio in the corresponding flux tube at $1 \mathrm{AU}$. The results found heretofore indicate a weak anticorrelation, which could be interpreted as the consequence of somewhat enhanced EUV fluxes related to interstream solar wind emanating from the fringes of closed field regions. The other approach involves a three-element correlation, using helium, hydrogen, and neon fluxes. Considering the more favorable Coulomb drag factors of $\mathrm{Ne}^{8+}$ compared to ${ }^{4} \mathrm{He}^{++}$, one expects the $\mathrm{Ne} / \mathrm{H}$ ratio to remain practically unaffected in the upper transition zone and the low corona. The $\mathrm{Ne} / \mathrm{H}$ ratio in the solar wind could therefore be used as a monitor for fractionating effects in the ionization process. The experimental evidence is ambiguous. Inspecting data from the ACE spacecraft one obtains the impression that the $\mathrm{Ne} / \mathrm{H}$ and the $\mathrm{He} / \mathrm{H}$ ratios in long-time averages correlate strongly, i.e., that the large variability of the $\mathrm{He} / \mathrm{H}$ ratio is correlated to a variability of the $\mathrm{Ne} / \mathrm{H}$ ratio of comparable importance. Either this could be the result of a variable FIP depletion of the two high-FIP elements $\mathrm{He}$ and $\mathrm{Ne}$, or this could be the consequence of inefficient Coulomb drag in a temperature region, where neon is still in a low ionization state. We will further comment on this possibility in the next paragraph.

Wimmer-Schweingruber (1994) has carried out a systematic investigation of interplanetary current-sheet-crossing events with Ulysses/SWICS data and found a very weak depletion of the He/O-ratio (of the order of 30\%) compared to coronal-hole-associated wind. Oxygen has a very similar first ionization potential as hydrogen, and the first ionization of oxygen strongly couples to the ionization of hydrogen through charge exchange between the two species. Hence, no strong fractionation through ionization is expected. Indeed, the $\mathrm{O} / \mathrm{H}$ ratio near current-sheet crossings remains virtually unchanged (WimmerSchweingruber 1994). Similarly, He/Ne-abundance ratios observed with ACE/SWICS reveal no trend while the $\mathrm{He} / \mathrm{H}$ ratio varies over a factor of 5 . This renders a general $\mathrm{He}$ depletion against $\mathrm{Ne}$ (and $\mathrm{H}$ ) due to inefficient ionization unlikely - a conclusion, which is reinforced when considering the photoionization cross sections shown in Fig. 11. Although neon has a somewhat lower ionization threshold, it seems extremely implausible that a variable ionizing spectrum is capable of separating helium and neon with variable fractionation factors.

Inefficient Coulomb drag remains an attractive option also to explain the variability of the $\mathrm{He} / \mathrm{Ne}$-abundance ratio as observed with the Apollo foils. Using the Coulomb fractionation model of Bodmer and Bochsler (1998), it is possible to correlate fractionation factors as follows:

$$
f_{i, j}=\frac{\left[1-C_{\mathrm{p}}^{*} H_{i} / \Phi_{\mathrm{p}}\right]}{\left[1-C_{\mathrm{p}}^{*} H_{j} / \Phi_{\mathrm{p}}\right]} .
$$




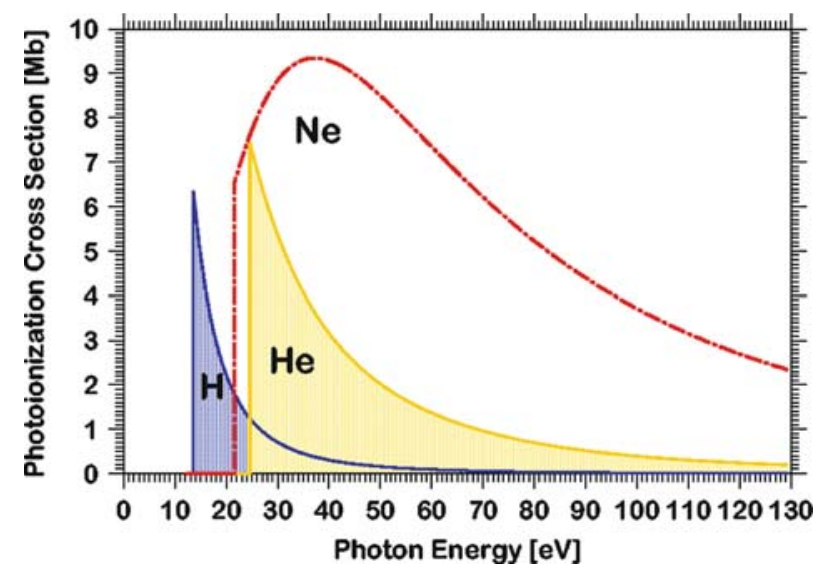

Fig. 11 Photoionization cross sections of H, He, and Ne according to Verner et al. (1996)

The fractionation factors are defined as the ratio of a measured isotopic abundance ratio divided by the corresponding ratio at the source (denoted with subscript o):

$$
f_{i, j}=: \frac{([i] /[j])}{([i] /[j])_{\mathrm{o}}} .
$$

$\Phi_{\mathrm{p}}$ in (17) is the proton flux integrated over the full space $\left(\Phi_{\mathrm{p}}=n v_{\mathrm{p}} \pi r^{2}\right)$ in units of $\mathrm{s}^{-1}$, and $\mathrm{C}_{\mathrm{p}}^{*}$ is a numerical factor, also in units of $\mathrm{s}^{-1}$, which relates the electrostatic interaction of the minor species with protons to the solar gravitational attraction (see Bochsler (2000) for details). $H_{i}$ is the Coulomb drag factor for the species $i$ :

$$
H_{i}=\frac{2 A-Q-1}{Q^{2}} \sqrt{\frac{A+1}{A}} .
$$

$A$ is again the mass of the particle in atomic units, and $Q$ is its effective charge in units of proton charges. Now, relating the elemental fractionation factor of helium with respect to neon, $f_{4,20}$, to the isotopic ${ }^{3} \mathrm{He} /{ }^{4} \mathrm{He}$-fractionation factor, $f_{4,3}$, it is possible to eliminate the ratio $\mathrm{C}_{\mathrm{p}}^{*} / \Phi_{\mathrm{p}}$ in (17):

$$
f_{4,20}=\frac{f_{4,3}\left(H_{4}-H_{3}\right)}{\left(H_{4}-H_{20}\right)-f_{4,3}\left(H_{3}-H_{20}\right)} \text {. }
$$

This is to be converted into abundance ratios with the above definitions of $f_{i, j}$

$$
([4] /[20])=\frac{\left(H_{4}-H_{3}\right) \cdot([4] /[3]) \cdot \frac{([4] /[20])_{o}}{([4] /[3])_{o}}}{\left(H_{4}-H_{20}\right)-\frac{([4] /[3])}{([4] /[3])_{o}} \cdot\left(H_{3}-H_{20}\right)} .
$$


In order to compare this simple model of Coulomb fractionation with observational data, one first needs to determine the only free parameters, which are the abundance ratios $([4] /[3])_{0}$ and $([4] /[20])_{0}$ at the source, i.e., in the photosphere. Since these are unknown, we have to find some other point along the dashed fractionation line in Fig. 12. We use the averages obtained with the Apollo-foil experiments Geiss et al. (2004) $([4] /[3])_{\mathrm{o}}=2,350$ and $([4] /[20])_{\mathrm{o}}=$ 570. The result of this comparison is shown in Fig. 12 with the dashed line delineating Eq. 21.

Another indication against atomic fractionation effects and against inefficient Coulomb-drag effects in low ionization states is the flatness and invariance of the low-FIP plateau from $\mathrm{Na}$ through $\mathrm{Ni}$ in low-speed as well as in coronal-hole-associated solar-wind regimes. We interpret this as strong evidence against drag-related fractionation among weakly ionizing species.

Table 4 is a summary of the discussion in this section. Assuming that just two different mechanisms, the so-called FIP effect and inefficient Coulomb drag (combined with gravitational deceleration), contribute to the shaping of coronal and solar-wind abundances, we conclude that - considering the observational evidence - the FIP effect plays a minor role for varying the $\mathrm{He} / \mathrm{H}$-abundance ratio. Most of it must be ascribed to the action of Coulomb drag. This bears consequences for the assessment of possible isotopic fractionation-effects in the solar wind.

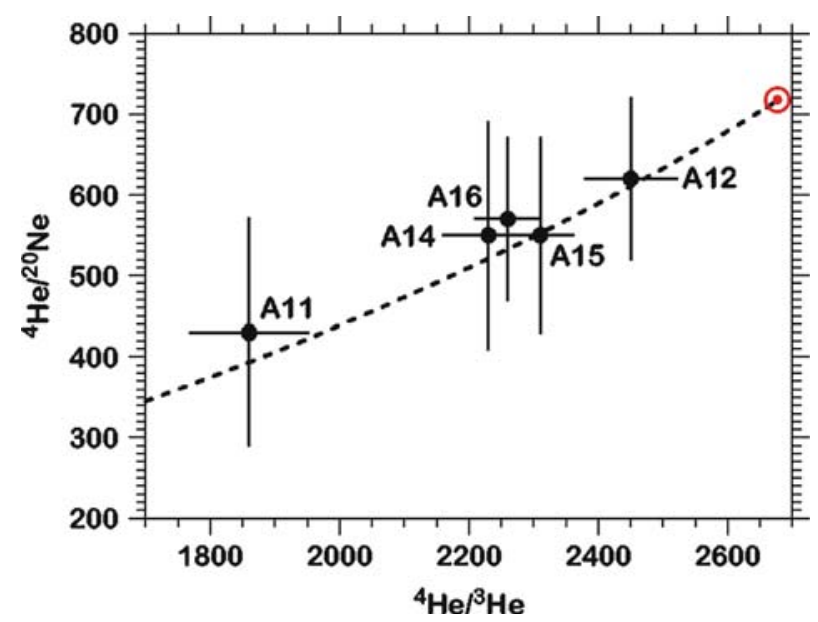

Fig. 12 The data points represent measurements of the individual Apollo foils as reported by Geiss et al. (1972, 2004). The dashed line illustrates the relation between the He/Ne-elemental abundance ratio and the ${ }^{4} \mathrm{He} /{ }^{3} \mathrm{He}$-isotopic ratio as predicted from the Coulomb-fractionation model (Eq. 21). Although the experimental uncertainties are too large to be conclusive, the agreement indicates that Coulomb fractionation is sufficient to explain the variations of the $\mathrm{He} / \mathrm{Ne}$-abundance ratios as observed with the Apollo foils, and no FIP fractionation needs to be invoked to explain these variations. To define a photospheric helium/neon-abundance ratio at the solar symbol on the fractionation line, the photospheric helium isotopic ratio ${ }^{4} \mathrm{He} /{ }^{3} \mathrm{He}=2,670$, derived by Bodmer and Bochsler (1998) (see Sect. 6.3) has been adopted 
Table 4 Elemental fractionation effects and causes

\begin{tabular}{|c|c|c|}
\hline Elemental ratio & $\begin{array}{l}\text { Possible effect by } \\
\text { FIP }\end{array}$ & $\begin{array}{l}\text { Possible effect by } \\
\text { inefficient Coulomb drag at high } \\
\text { temperature and low density }\end{array}$ \\
\hline $\mathrm{He} / \mathrm{H}$ & Weak & Strong \\
\hline $\mathrm{O} / \mathrm{H}$ & None & Weak \\
\hline $\mathrm{Ne} / \mathrm{H}$ & Weak & Weak \\
\hline $\mathrm{He} / \mathrm{O}$ & Weak & Moderate \\
\hline $\mathrm{He} / \mathrm{Ne}$ & None (see Fig. 11) & Moderate (see Fig. 12) \\
\hline $\mathrm{Na} / \mathrm{Fe}$ & None & Weak or none \\
\hline
\end{tabular}

\subsection{Some inferences from solar-wind isotopic abundances}

Based on the SWICS/Ulysses measurements in the solar wind and assuming that the systematic depletion of the helium abundance in the solar wind was entirely due to inefficient Coulomb drag, Bodmer and Bochsler (1998), derived a ${ }^{4} \mathrm{He}:{ }^{3} \mathrm{He}$ ratio of 2,670 \pm 500 for the OCZ, which corresponds to ${ }^{3}(\mathrm{He} / \mathrm{H})_{\mathrm{OCZ}}=(3.10 \pm 0.66) \times 10^{-5}$. Assuming an initial ${ }^{3} \mathrm{He}$ abundance adopted from the Jovian atmosphere (Mahaffy et al. 2000) and from the solar $\mathrm{He} / \mathrm{H}$-abundance ratio, yields ${ }^{3} \mathrm{He} / \mathrm{H}_{\text {initial }}=1.38 \times 10^{-5}$. Furthermore, assuming an initial $\mathrm{D} / \mathrm{H}$ ratio, as presently observed in the interstellar medium, as a lower limit for the presolar deuterium abundance, $\mathrm{D} / \mathrm{H}_{\text {initial }} \leq 1.5 \times 10^{-5}$, leaves for a possible secular increase less than $2 \mathrm{ppm}$ of ${ }^{3} \mathrm{He} / \mathrm{H}$, which could be attributed to leakage from the radiative zone into the OCZ.

The fact that the ${ }^{3} \mathrm{He} / \mathrm{H}$ ratio amounts to as much as $2 \%$ at the fringes of the nuclear burning zone near the mass coordinate $M_{r} \simeq 0.6 M_{\odot}$ shows that any mixing or diffusive process is extremely weak. Turbulent mixing of the Sun has been considered in different scenarios as a remedy for the neutrino problem in the past few decades (Schatzmann 1969). Note that the absence of a noticeable "3 He-contamination" in the solar atmosphere not only eliminates the operation of a long-time process but also the possibility of periodic occurrences of short-time mixing, since such a diagnostic has a memory over the entire solar lifetime, not to be erased in the OCZ.

Constraining fractionation by inefficient Coulomb-drag with the solar-wind $\mathrm{He} / \mathrm{H}$-depletion, and using the ${ }^{20} \mathrm{Ne} /{ }^{22} \mathrm{Ne}$ solar-wind values provided with the Apollo foils (Geiss et al. 1972) and with the SOHO/CELIAS experiment Kallenbach et al. (1997), Wiens et al. (2004) estimated the photospheric ${ }^{20} \mathrm{Ne} / 22 \mathrm{Ne}$ ratio to be $13.35 \pm 0.80$. Of course, this information is of limited use, since there is no comparable value for the interstellar medium. Nevertheless, it might serve as a baseline for models of evolution of planetary and cometary atmospheres and of fractionation within the solar system and the heliosphere, as constraint for galactic chemical evolution models, or as benchmark for nucleosynthetic modeling of stellar evolution.

Similarly, on the basis of the Coulomb-fractionation model and of Fig. 12 we can derive a He/Ne-elemental abundance ratio for the OCZ by combining 
the Apollo-foil measurements and the Coulomb-fractionation theory. We estimate the ratio for $\left({ }^{4} \mathrm{He} /{ }^{20} \mathrm{Ne}\right)_{\mathrm{OCZ}}$ to be $720 \pm 200$. Together with the isotopic abundances derived above, this yields

$$
(\mathrm{He} / \mathrm{Ne})_{\mathrm{OCZ}}=670 \pm 200 .
$$

Provided that the model assumptions concerning Coulomb-drag fractionation are correct, we are now in a situation to derive a neon abundance for the OCZ, which has the advantage of being directly related to a helioseismological determination of helium and being absolutely independent of photospheric abundance determinations. Adopting the value $Y=0.247$ used by Däppen (1998), we find a logarithmic dex value of 10.92, which brings the solar neon abundance $A(\mathrm{Ne})$ to $8.09 \pm 0.15$, ( $2 \sigma$ uncertainty) consistent with the value reported by Holweger (2001) (see the entry for neon in Table 2). Our value is, however, incompatible with the value $A(\mathrm{Ne})=8.29 \pm 0.05$ used by Bahcall et al. (2005) as a remedy for the solar opacity problem after the revision of photospheric abundances by Asplund et al. (2005).

Combining our result for the solar neon-abundance with the result of the neon/oxygen-abundance ratio as shown in Table 1 we derive the solar oxygen abundance to be $A(\mathrm{O})=8.94 \pm 0.15$. This determination rests on the assumption that the solar wind faithfully reflects the solar neon/oxygen-abundance ratio. This seems justified in view of the fact (see Sect. 6.2) that neon and oxygen are both high-FIP elements with neighboring masses, which maintain a constant abundance ratio under different solar-wind conditions (Bochsler et al. 1986, von Steiger et al. 2000). Furthermore, oxygen- and neon-abundances are generally strongly correlated in the solar wind despite concomitant large $\mathrm{He} / \mathrm{H}$ variations. Note that our value is, again, independent of photospheric abundance determinations since it rests entirely on solar-wind measurements and the helioseismological determination of helium in the OCZ. It is consistent with the older photospheric determination of Grevesse and Sauval (1998) but incompatible with the revised values of Asplund et al. (2005).

Several other applications for solar-system research and for galactic chemical evolution, similar to those listed above, will evolve, once the interpretation of the Genesis solar-wind sample return mission will become available. However, it is important to note that valid inferences for the solar isotopic composition from solar-wind measurements will require further careful investigation of the variability of solar-wind isotopic fractionation effects. Furthermore, the isotopic fractionation effect of gravitational settling through the lower boundary of the OCZ needs to be assessed carefully for this type of applications (Bochsler 2000; Turcotte and Wimmer-Schweingruber 2002).

\section{Conclusions}

In this review we have dealt with various aspects of minor species in the solar wind. It is interesting to note that although it was realized early on that the 
solar wind not only consists of protons and electrons, it took about 20 years to recognize that helium, which makes up only about $5 \%$ in numbers, plays an important and active role in the dynamics of solar wind (Noci and Porri 1983, Bürgi and Geiss 1986). Throughout this review, we have not included a discussion of pickup ions, which also play an important role in the dynamics of the solar wind as one moves beyond 10 AU away from the Sun. Pickup ions originating from interstellar gas and presumably from the interaction of interstellar dust with solar-wind ions populate the energy ranges outside those of the typical solar wind even in the innermost part of the solar system. They act as seeds for solar energetic particles and they are specially susceptible to acceleration by interplanetary shocks and coronal mass ejections. The so-called anomalous cosmic-ray component is also thought to originate from pickup ions (Fisk et al. 1974).

Realizing that the solar wind is recorded in lunar and asteroidal regoliths, helped in the early history of solar-wind research to develop experimental methods and theoretical concepts for the understanding of minor species in the solar wind. It was planned to incorporate a section on the historic solar wind into this review. The possibility to use regoliths as archives for the history of the solar wind and the near-Earth environment during the past billion years has been promoted by different authors at many occasions (see Kim et al. (1995) for an illustrative example). However, this now seems premature, given the recent lessons of the Genesis mission, which have shown, that deciphering these archives is not as simple as previously thought. Several findings attributed to a secular variability of the solar wind are now suspected to be due to biases of implantation processes and storage of low-energy particles in solids. Despite all these difficulties, it seems clear that the irradiation history of regoliths has been linked with well-identifiable time marks, and the potential of documenting space climate in the past, remains intact, even if the scripture is more difficult to read than previously anticipated.

Acknowledgments This review is dedicated to the memory of Hartmut Holweger, who passed away on April 5, 2006 and who has significantly contributed to the foundation of solar abundances. Much of the discussion on abundances in the solar wind rests on his work. The author enjoyed scientific enlightening and took benefit from many discussions during his stay at the University of New Hampshire particularly with Eberhard Möbius, Harald Kucharek, Martin Lee, and Phil Isenberg. He acknowledges helpful discussions with Fred Ipavich, Manfred Scholer, Ansgar Grimberg and Fritz Bühler. The author also wishes to express his gratitude to Martin C.E. Huber, who patiently worked through the manuscript contributing numerous improvements. This work was partly supported by the Swiss National Science Foundation and by NASA through grant NAG5-12929.

\section{References}

Abbas MM, LeClair A, Owen T, Conrath BJ, Flasar FM, Kunde VG, Nixon CA, Achterberg RK, Bjoraker G, Jennings DJ, Orton G, Romani PN (2004) The nitrogen isotopic ratio in Jupiter's atmosphere from observations by the composite infrared spectrometer on the Cassini spacecraft. Astrophys J 602:1063-1074

Aellig MR, Grünwaldt H, Bochsler P, Hefti S, Wurz P, Kallenbach R, Ipavich FM, Hilchenbach M, the CELIAS Team (1997a) Solar wind minor ion charge states observed with high 
time resolution with SOHO/CELIAS/CTOF. In: Proceedings of 31st ESLAB-symposium 'Correlated Phenomena at the Sun, in the Heliosphere and in Geospace', ESTEC ESA SP-415, Noordwijk, The Netherlands, pp 27-31

Aellig MR, Grünwaldt H, Bochsler P, Wurz P, Hefti S, Kallenbach R, Ipavich FM, Hovestadt D, Hilchenbach M, the CELIAS Team (1997b) Solar wind iron charge states observed with high time resolution with SOHO/CELIAS/CTOF. In: Proceedings of the 5th SOHO workshop: 'The corona and solar wind near minimum activity'. Oslo, Norway, ESTEC ESA SP-404, Noordwijk, The Netherlands, pp 157-161

Aellig MR, Bochsler P, Grünwaldt H, Hefti S, Wurz P, Hilchenbach M, Hovestadt D, Ipavich FM, Gliem F (1998) The influence of suprathermal electrons on the derivation of coronal electron temperatures from solar wind minor ion charge states. Phys Chem Earth 24:407-414

Aellig MR, Hefti S, Grünwaldt H, Bochsler P, Wurz P, Ipavich FM, Hovestadt D (1999a) The $\mathrm{Fe} / \mathrm{O}$ elemental abundance ratio in the solar wind as observed with SOHO/CELIAS/CTOF. J Geophys Res 104:24769-24780

Aellig MR, Holweger H, Bochsler P, Wurz P, Grünwaldt H, Hefti S, Ipavich FM, Klecker B (1999b) The Fe/O elemental abundance ratio in the solar wind. In: Habbal SR, Esser R, Hollweg JV, Isenberg PA (eds) Solar wind nine. AIP Proceedings 471, pp 255-258

Arnaud M, Raymond J (1992) Iron ionization and recombination and ionization equilibrium. Astrophys J 398:394-406

Arnaud M, Rothenflug R (1985) An updated evaluation of recombination and ionization rates. Astron Astrophys Suppl Ser 60:425-457

Asbridge JR, Bame SJ, Feldman WC, Montgomery MD (1976) Helium and hydrogen velocity differences in the solar wind. J Geophys Res 81(16):2719-2727

Asplund M, Grevesse N, Sauval AJ (2005) The solar chemical composition. In: Barnes TG, Bash FN (eds) ASP Conf. Ser. 336: cosmic abundances as records of stellar evolution and nucleosynthesis, p 25

Bahcall JN, Basu S, Serenelli AM (2005) What is the neon abundance of the sun? Astrophys J 631:1281-1285

Bame SJ, Hundhausen AJ, Asbridge JR, Strong IB (1968) Solar wind ion composition. Phys Rev Lett 20:393-395

Bird M, Edenhofer P (1990) Remote sensing observations of the solar corona, vol 1 Chap 2. Springer, Berlin Heidelberg New York, pp 13-97

Bochsler P (1984) Helium and oxygen in the solar wind: dynamic properties and abundances of elements and helium isotopes as observed with the ISEE-3 plasma composition experiment Habilitationsschrift, University of Bern

Bochsler P (1989) Velocity and abundances of silicon ions in the solar wind. J Geophys Res 94:23652373

Bochsler P (2000) Abundances and charge states of particles in the solar wind. Rev Geophys 38(2):247-266

Bochsler P, von Steiger R (2004) Inferences for isotopic fractionation processes in the solar wind using the full solar cycle record of abundances from Ulysses: Anticipating results from the Genesis mission. In: Proceedings of the SOHO 15 workshop on coronal heating, ESA SP-575, pp 372-377

Bochsler P, Geiss J, Kunz S (1986) Abundances of carbon, oxygen and neon in the solar wind during the period from August 1978 to June 1982. Solar Phys 103:177-201

Bochsler P, Gonin M, Sheldon RB, Zurbuchen T, Gloeckler G, Hamilton DC, Collier MR, Hovestadt D (1996) Abundance of solar wind magnesium isotopes determined with WIND/MASS. In: Winterhalter D, Gosling JT, Habbal SR, Kurth WS, Neugebauer M (eds) Solar wind eight. Proceedings of the 8th international solar wind conference, vol 382, American Institute of Physics, Woodbury, NY, USA, pp 199-202

Bochsler P, Ipavich FM, Paquette JA, Weygand JM, Wurz P (2000) Determination of the abundance of aluminum in the solar wind with SOHO/CELIAS/MTOF. J Geophys Res 105:12,659-12,666

Bodmer R, Bochsler P (1998) The helium isotopic ratio in the solar wind and ion fractionation in the corona by inefficient coulomb drag. Astron Astrophys 337:921-927

Bürgi A, Geiss J (1986) Helium and minor ions in the corona and solar wind: dynamics and charge states. Solar Phys 103:347-383 
Carlsson M, Stein RF (1999) The dynamic solar chromosphere and the ionization of hydrogen. In: Habbal SR, Esser R, Hollweg JV, Isenberg PA (eds) Solar wind nine. AIP proceedings 471, pp 23-28

Carlsson M, Stein RF (2002) Dynamic hydrogen ionization. Astrophys J 572:626-635

Cerutti H (1974) Die Bestimmung des Argons im Sonnenwind aus Messungen an den ApolloSWC-Folien. PhD thesis, University of Bern, Switzerland

Cohen CMS, Leske RA, Christian ER, Cummings AC, Mewaldt RA, Slocum PL, Stone EC, von Rosenvinge TT, Wiedenbeck ME (2000) The isotopic composition of solar energetic particles. In: Mewaldt RA, Jokipii JR, Lee MA, Möbius E, Zurbuchen TH (eds) Acceleration and transport of energetic particles observed in the heliosphere. AIP proceedings 528, pp 55-62

Collier MR, Hamilton DC, Gloeckler G, Bochsler P, Sheldon RB (1996) Neon-20, oxygen-16 and helium-4 densities, temperatures, and suprathermal tails in the solar wind determined with WIND/MASS. Geophys Res Lett 23:1191-1194

Collier MR, Hamilton DC, Gloeckler G, Ho G, Bochsler P, Bodmer R, Sheldon R (1998) Oxygen 16 to oxygen 18 abundance ratio in the solar wind observed by Wind/MASS. J Geophys Res 103:7-13

Coplan MA, Ogilvie KW, Bochsler P, Geiss J (1978) Ion composition experiment. IEEE Trans Geosci Electron GE-16(3):185-191

Coplan MA, Ogilvie KW, Bochsler P, Geiss J (1984) Interpretation of ${ }^{3} \mathrm{He}$ abundance variations in the solar wind. Solar Phys 93:415-434

Cranmer SR (2002) Coronal holes and the high-speed solar wind. Space Sci Rev 101:229-294

Cranmer SR, Field GB, Kohl JL (1999a) The impact of ion-cyclotron wave dissipation on heating and accelerating the fast solar wind. In: Habbal SR, Esser R, Hollweg JV, Isenberg PA (eds) Solar wind nine. AIP proceedings 471, pp 35-38

Cranmer SR, Field GB, Kohl JL (1999b) Spectroscopic constraints on models of ion cyclotron resonance heating in the polar solar corona and high-speed solar wind. Astrophys J 518:937-947

Däppen W (1998) Microphysics: equation of state. Space Sci Rev 85:49-60

Dzifčáková E (1998) The ionization equilibrium in the solar corona for the electron power distribution. Solar Phys 178:317-339

Dzifčáková E (2000) Electron excitation rates in the solar corona for non-Maxwellian electron distributions. Solar Phys 196:113-127

Eberhardt P, Geiss J, Grögler N (1966) Distribution of rare gases in the pyroxene and feldspar of the Khor Temiki meteorite. Earth Planet Sci Lett 1:7-12

Eberhardt P, Geiss J, Graf H, Grögler N, Krähenbühl U, Schwaller H, Schwarzmüller J (1970) Trapped solar wind noble gases, exposure age and K/Ar-age in Apollo 11 lunar fine material. Proc Apollo 11 Lunar Sci Conf 2:1037-1070

Fisk LA, Kozlovsky B, Ramaty R (1974) An interpretation of the observed oxygen and nitrogen enhancements in low-energy cosmic rays. Astrophys J 190:L35-L37

Galvin AB, Ipavich FM, Gloeckler G, von Steiger R, Wilken B (1992) Silicon and oxygen charge state distributions and relative abundances in the solar wind measured by SWICS on Ulysses. In: Marsch E, Schwenn R (eds) Solar Wind Seven. Proceedings of the 3rd COSPAR Colloquium held in Goslar, Germany 1991, pp 337-340

Geiss J, Bochsler P (1985) Ion composition in the solar wind in relation to solar abundances. In: Rapports isotopiques dans le système solaire, Cépaduès Editions, pp 213-228

Geiss J, Hirt P, Leutwyler H (1970) On acceleration and motion of ions in corona and solar wind. Solar Phys 12:458-483

Geiss J, Bühler F, Cerutti H, Eberhardt P, Filleux C (1972) Solar wind composition experiment. Apollo 16 Prel Sci Rep NASA Spec Publ 315(14):14.1-14.10

Geiss J, Gloeckler G, von Steiger R (1995) Origin of the solar wind from composition data. Space Sci Rev 72:49-60

Geiss J, Bühler F, Cerutti H, Eberhardt P, Filleux C, Meister J, Signer P (2004) The Apollo SWC experiment: results, conclusions, consequences. Space Sci Rev 110:307-335

Gloeckler G, Geiss J (1998) Measurement of the abundance of helium-3 in the Sun and in the local interstellar cloud with SWICS on Ulysses. Space Sci Rev 84:275-284

Gloeckler G, Geiss J, Balsiger H, Fisk LA, Gliem F, Ipavich FM, Ogilvie KW, Stüdemann W, Wilken B (1983) The ISPM solar-wind ion composition spectrometer. ESA Special Publ SP-1050: $75-103$ 
Gloeckler G, Ipavich FM, Hamilton DC, Wilken B, Stüdemann W, Kremser G (1986) Solar wind carbon, nitrogen and oxygen abundances measured in the Earth's magnetosheath with AMPTE/CCE. Geophys Res Lett 13:793-796

Gosling JT, Asbridge J, Bame SJ, Feldman WC, Zwickl RD (1980) Observations of large fluxes of $\mathrm{He}^{+}$in the solar wind following an interplanetary shock. J Geophys Res 85(A7):3431-3434

Grevesse N, Sauval AJ (1998) Standard solar composition. Space Sci Rev 85:161-174

Gringauz KI, Bezrukikh VV, Ozerov VD, Ribchinsky RE (1960) Study of the interplanetary high energy electrons, and corpuscular radiation by means of three electrode traps for charged particles on the second soviet cosmic rocket. Soviet Phys Dokl 53:61

Gringauz KI, Bezrukikh VV, Ozerov VD, Ribchinsky RE (1961) Some results of experiments in interplanetary space by means of charged particle traps on soviet space probes. Space Res 2:539-553

Hefti S, Grünwaldt H, Ipavich FM, Bochsler P, Hovestadt D, Aellig MR, Hilchenbach M, Kallenbach R, Galvin AB, Geiss J, Gliem F, Gloeckler G, Klecker B, Marsch E, Möbius E, Neugebauer M, Wurz P (1998) Kinetic properties of solar wind minor ions and protons measured with SOHO/CELIAS. J Geophys Res 103:29697-29704

Ho GC (1998) Helium-3 enhancements and unusual ion charge state composition in coronal mass ejections. $\mathrm{PhD}$ thesis, University of Maryland

Ho GC, Hamilton DC, Gloeckler G, Bochsler P (2000) Enhanced solar wind 3He2+ associated with coronal mass ejections. Geophys Res Lett 27: 309-312

Hollweg JV (1974) Alfvénic acceleration of solar wind helium and related phenomena. J Geophys Res 79(10):1357-1363

Holweger H (2001) Photospheric abundances: Problems, updates, implications. In: Wimmer-Schweingruber RF (ed) Solar and galactic composition. AIP conference proceedings, Melville, NY, vol CP-598, pp 23-30

Hovestadt D, Hilchenbach M, Bürgi A, Klecker B, Laeverenz P, Scholer M, Grünwaldt H, Axford WI, Livi S, Marsch E, Wilken B, Winterhoff HP, Ipavich FM, Bedini P, Coplan MA, Galvin AB, Gloeckler G, Bochsler P, Balsiger H, Fischer J, Geiss J, Kallenbach R, Wurz P, Reiche KU, Gliem F, Judge DL, Ogawa HS, Hsieh KC, Möbius E, Lee MA, Managadze GG, Verigin MI, Neugebauer M (1995) CELIAS - Charge, element and isotope analysis system for SOHO. Solar Phys 162:441-481

Hufbauer K (1991) Exploring the Sun: solar science since Galileo. Johns Hopkins University Press, Baltimore

Hundhausen AJ, Gilbert HE, Bame SJ (1968a) Ionization state of the interplanetary plasma. J Geophys Res 73:5485-5493

Hundhausen AJ, Gilbert HE, Bame SJ (1968b) The state of ionization of oxygen in the solar wind. Astrophys J 152:L3-L5

Ipavich FM (2006) Preliminary, personal communication

Ipavich FM, Bochsler P, Lasley SE, Paquette JE, Wurz P (1999) The abundance of sodium in the solar wind as measured by SOHO/CELIAS/MTOF. EOS Trans AGU 80:256

Ipavich FM, Paquette JA, Bochsler P, Lasley SE, Wurz P (2001) Solar wind iron isotopic abundances: Results from SOHO/CELIAS/MTOF. In: Wimmer-Schweingruber RF (ed) Solar and galactic composition. AIP conference proceedings, Melville, NY, vol CP-598, pp 121-126

Isenberg P, Hollweg JV (1982) Finite amplitude Alfvén waves in a multi-ion plasma: Propagation, acceleration and heating. J Geophys Res 87:5023-5029

Kallenbach R, Ipavich FM, Bochsler P, Hefti S, Hovestadt D, Grünwaldt H, Hilchenbach M, Axford WI, Balsiger H, Bürgi A, Coplan MA, Galvin AB, Geiss J, Gliem F, Gloeckler G, Hsieh K, Lie MA, Livi S, Managadze GG, Marsch E, Möbius E, Neugebauer M, Reiche KU, Scholer M, Verigin MI, Wilken B, Wurz P (1997) Isotopic composition of solar wind neon measured by CELIAS/MTOF on board SOHO. J Geophys Res 102:26895-26904

Kallenbach R, Geiss J, Ipavich FM, Gloeckler G, Bochsler P, Gliem F, Hefti S, Hilchenbach M, Hovestadt D (1998a) Isotopic composition of solar wind nitrogen: First in situ determination with CELIAS/MTOF spectrometer on board SOHO. Astrophys J 507:L185-L188

Kallenbach R, Ipavich FM, Bochsler P, Hefti S, Wurz P, Aellig MR, Coplan MA, Galvin AB, Gloeckler G, Grünwaldt H, Hilchenbach M, Hovestadt D, Klecker B, Reiche KU, the CELIAS Team (1998b) Isotopic composition of solar wind calcium measured by CELIAS/MTOF on board SOHO. Astrophys J 498:L75-L78 
Kallenbach R, Ipavich FM, Kucharek H, Bochsler P, Galvin AB, Geiss J, Gliem F, Gloeckler G, Grünwaldt H, Hefti S, Hilchenbach M, Hovestadt D (1998c) Fractionation of Si, Ne, and Mg isotopes in the solar wind as measured by SOHO/CELIAS/MTOF. Space Sci Rev 85:357-370

Kern O (1999) Abundances and charge-state composition of calcium, silicon, and iron in the solar wind measured with the WIND/MASS sensor. PhD thesis, University of Bern, Switzerland

Kern O, Wimmer-Schweingruber RF, Bochsler P, Gloeckler G, Hamilton DC (1997) Determination of calcium and silicon charge states and elemental abundances in the solar wind with the MASS instrument on WIND. In: Proceedings of the 31st ESLAB symposium, 'Correlated Phenomena at the Sun, in the Heliosphere and in Geospace'. Workshop on plasma dynamics and diagnostics in the solar transition region and corona, ESA SP-415, pp 345-348

Kim J, Kim Y, Marti K, Kerridge JF (1995) Nitrogen isotope abundances in the recent solar wind. Nature 375(6530):383-385

Ko YK, Fisk LA, Gloeckler G, Geiss J (1996) Limitations on suprathermal tails of electrons in the lower solar corona. Geophys Res Lett 23:2785-2788

Kohl J, Esser R, Gardner LD, Habbal S, Daigneau PS, Dennys EF, Nystrom GU, Panasyuk A, Raymond JC, Smith PL, Strachan L, van Ballegooijen AA, Noci G, Fineschi S, Romoli M, Ciaravella A, Modigliani A, Huber MCE, Antonucci E, Benna C, Giordano S, Tondello G, Nicolosi P, Naletto G, Spadaro D, Poletto G, Livi S, von der Lühe O, Geiss J, Timothy JG, Gloeckler G, Allegra A, Basile G, Brusa R, Wood B, Siegmund OHW, Fowler W, Fisher R, Habvala J (1995) The ultraviolet coronagraph spectrometer for the solar and Heliospheric observatory. Solar Phys 162:313-356

Kohl JL, Noci G, Antonucci E, Tondello G, Huber MCE, Gardner LD, Nicolosi P, Strachan L, Fineschi S, Raymond JC, Romoli M, Spadaro D, Panasyuk A, Siegmund OHW, Benna C, Ciaravella A, Cranmer SR, Giordano S, Karovska M, Martin R, Michels J, Modigliani A, Naletto G, Pernechele C, Poletto G, Smith PL (1997) First results from the SOHO ultraviolet coronagraph spectrometer. Solar Phys 175(2):613-644

Kohl JL, Noci G, Cranmer SR, Raymond JC (2006) Ultraviolet spectroscopy of the extended solar corona. Astron Astrophys Rev. DOI 10.1007/s00,159-005-0026-7

Kucharek H, Ipavich FM, Kallenbach R, Bochsler P, Hovestadt D, Grünwaldt H, Hilchenbach M, Axford WI, Balsiger H, Bürgi A, Coplan MA, Galvin AB, Geiss J, Gliem F, Gloeckler G, Hsieh K, Klecker B, Lee MA, Livi S, Managadze GG, Marsch E, Möbius E, Neugebauer M, Reiche KU, Scholer M, Verigin MI, Wilken B, Wurz P (1998) Magnesium isotopic composition as observed with the MTOF sensor of the CELIAS experiment on the SOHO spacecraft. J Geophys Res 103:26805-26812

Lee T, Papanastassiou D, Wasserburg GJ (1976) Demonstration of ${ }^{26} \mathrm{Mg}$ excess in allende and evidence for ${ }^{26} \mathrm{Al}$. Geophys Res Lett 3:41-44

Leske RA, Mewaldt RA, Cohen CMS, Christian ER, Cummings AC, Slocum PL, Stone EC, von Rosenvinge TT, Wiedenbeck ME (2001) Isotopic abundances in the solar corona as inferred from ACE measurements of solar energetic particles. In: Wimmer-Schweingruber RF (ed) Solar and galactic composition, AIP conference proceedings, Melville, NY, vol CP-598, pp 127-132

Leutwyler H (1999) Personal communication

Livi S, Marsch E (1987) Generation of solar wind proton tails and double beams by Coulomb collisions. J Geophys Res 92(A7):7255-7261

Mahaffy PR, Niemann HB, Alpert A, Atreya SK, Demick J, Donahue TM, Harpold DN, Owen TC (2000) Noble gas abundance and isotope ratios in the atmosphere of Jupiter from the Galileo Probe Mass Spectrometer. J Geophys Res 105:15061-15071

Marsch E, Mühlhäuser KH, Rosenbauer H, Schwenn R, Neubauer FM (1982) Solar wind helium ions: observations of the Helios solar probes between 0.3 and 1 AU. J Geophys Res 87:35-51

Marsch E, von Steiger R, Bochsler P (1995) Element fractionation by diffusion in the solar chromosphere. Astron Astrophys 301:261-276

McKenzie JF (1994) Interaction between Alfvén waves and a multicomponent plasma with differential ion streaming. J Geophys Res 99(A3):4193-4200

McKenzie JF, Ip WH, Axford WI (1979) The acceleration of minor ion species in the solar wind. Astrophys Space Sci 64:183-211

Neugebauer M (1997) A career in the solar wind. J Geophys Res 102:26,887-26,894

Neugebauer MM, Snyder CW (1962) The mission of Mariner II: Preliminary observations. Solar plasma experiment. Science 138:1095-1096 
Neugebauer MM, Snyder CW (1966) Mariner 2 observations of the solar wind. 1. Average properties. J Geophys Res 71:4469-4484

Neukomm RO, Bochsler P (1996) Diagnostics of closed magnetic structures in the solar corona using charge states of helium and of minor ions. Astrophys J 465:462-472

Noci G, Porri A (1983) Models of the solar wind acceleration region. IAGA, Hamburg, paper 4L.04 presented at the 18th General Assembly Meeting

Oetliker M, Hovestadt D, Klecker B, Collier MR, Gloeckler G, Hamilton DC, Ipavich FM, Bochsler P, Managadze GG (1997) The isotopic composition of iron in the solar wind: First measurements with the mass sensor on the WIND spacecraft. Astrophys J 474:L69-L79

Ogilvie KW (1975) Differences between the bulk speeds of hydrogen and helium in the solar wind. J Geophys Res 80:1335-1338

Ogilvie KW, Bochsler P, Geiss J, Coplan MA (1980a) Observations of the velocity distribution of solar wind ions. J Geophys Res 85:6069-6074

Ogilvie KW, Coplan MA, Bochsler P, Geiss J (1980b) Abundance ratios of ${ }^{4} \mathrm{He}^{++} \beta \mathrm{He}^{++}$in the solar wind. J Geophys Res 85:6021-6024

Owen T, Mahaffy PR, Niemann HB, Atreya S, Wong M (2001) Protosolar nitrogen. Astrophys J 553:L77-L79

Owocki SP, Scudder JD (1983) The effect of a non-maxwellian electron distribution on oxygen and iron ionization balances in the solar corona. Astrophys J 270:758-768

Owocki SP, Holzer TE, Hundhausen AJ (1983) The solar wind ionization state as a coronal temperature diagnostic. Astrophys J 275:354-366

Paquette JA, Ipavich FM, Lasley SE, Bochsler P, Wurz P (2001) The relative abundance of chromium and iron in the solar wind. In: Wimmer-Schweingruber RF (ed) Solar and galactic composition. AIP conference proceedings, Melville, NY, vol CP-598, pp 95-100

Parker EN (1958) Dynamics of the interplanetary gas and magnetic fields. Astrophys J 128:664-676

Parker EN (1961) The solar wind. McGraw-Hill, New York, pp 157-170

Parker EN (1965) Dynamical theory of the solar wind. Space Sci Rev 4: 666-708

Parker EN (2001) A history of the solar wind concept. In: Bleeker JAM, Geiss J, Huber MCE (eds) The century of space science vol 1. Kluwer Academic Dordrecht, pp 225-255

Porquet D, Arnaud M, Decourchelle A (2001) Impacts of a power-law non-thermal electron tail on the ionization and recombination rates. Astron Astrophys 373:1110-1124

Reames DV (1994) Coronal abundances derived from energetic particles. Adv Space Res 15(7): 41-51

Reisenfeld DB, Gary SP, Gosling JT, Steinberg JT, McComas DJ, Goldstein BE, Neugebauer M (2001) Helium energetics in the high-latitude solar wind: Ulysses observations. J Geophys Res 106(A4):5693-5708

Reisenfeld DB, Steinberg JT, Barraclough BL, Dors EE, Wiens RC, Neugebauer M, Reinard A, Zurbuchen T (2003) Comparison of the Genesis solar wind regime algorithm results with solar wind composition observed by ACE. In: Velli M, Bruno R, Malara F (eds) Solar wind ten. AIP conference proceedings CP679, pp 632-635

Richardson IG, Cane HV (2004) Identification of interplanetary coronal mass ejections at 1 au using multiple solar wind plasma composition anomalies. J Geophys Res 109(A09104):1-16

Rosenbauer H, Schwenn R, Marsch E, Meyer B, Miggenrieder H, Montgomery MD, Mühlhäuser KH, Pilipp W, Voges W, Zink SM (1977) A survey of initial results of the Helios plasma experiment. J Geophys Res 42:561-580

Schatzmann E (1969) Turbulent transport, solar lithium and solar neutrinos. Astrophys Lett 3 : $139-140$

Schmid J, Bochsler P, Geiss J (1987) Velocity of iron ions in the solar wind. J Geophys Res 92: 9901-9906

Schmid J, Bochsler P, Geiss J (1988) Abundance of iron ions in the solar wind. Astrophys J 329: 956-966

Schwadron NA, Fisk LA, Zurbuchen TH (1999) Elemental fractionation in the slow solar wind. Astrophys J 521:859-867

Schwadron NA, McComas DJ, Elliott HA, Gloeckler G, Geiss J, von Steiger R (2005) Solar wind from the coronal hole boundaries. J Geophys Res 110(A04140). DOI:10.1029/2004JA010,896

Schwenn R, Rosenbauer H, Mühlhäuser KH (1980) Singly-ionized helium in the driver gas of an interplanetary shock wave. Geophys Res Lett 7:201-204 
Spitzer L (1956) Physics of fully ionized gases. Interscience, New York

von Steiger R, Geiss J (1989) Supply of fractionated gases to the corona. Astron Astrophys 225: $222-238$

von Steiger R, Geiss J, Gloeckler G, Galvin AB (1995) Kinetic properties of heavy ions in the solar wind from SWICS/Ulysses. Space Sci Res 72:71-76

von Steiger R, Schwadron NA, Hefti S, Fisk LA, Geiss J, Gloeckler G, Wilken B, Wimmer-Schweingruber RF, Zurbuchen TH (2000) Composition of quasistationary solar wind flows from SWICS/Ulysses. J Geophys Res 105:27217-27238

Tu CY, Wang LH, Marsch E (2003) A possible way of understanding the differential motion of minor ions in the solar wind. J Geophys Res 108(A4):SSH9-1-SSH9-10

Turcotte S, Wimmer-Schweingruber RF (2002) Possible in situ tests of the evolution of elemental and isotopic abundances in the solar convection zone. J Geophys Res 107(A12):1-11

Verner DA, Ferland GJ, Korista KT, Yakovlev DG (1996) Atomic data for astrophysics. II. New analytic fits for photoionization cross sections of atoms and ions. Astrophys J 465:487-498

Voronov GS (1997) A practical fit formula for ionization rate coefficients of atoms and ions by electron impact: $\mathrm{Z}=1-28$. Atomic Data Nuclear Data Tables 65:1-35

Wänke H (1965) Der Sonnenwind als Quelle der Uredelgase in Steinmeteoriten. Z Naturforschg 20a:946-949

Webb DF, Howard RA (1994) The solar cycle variation of coronal mass ejections and the solar wind mass flux. J Geophys Res 99:4201-4220

Weygand JM, Ipavich FM, Wurz P, Paquette JA (2001a) Determination of the 36Ar/38Ar isotopic abundance ratio of the solar wind using SOHO/CELIAS/MTOF. Geochim Cosmochim Acta 65:4589-4596

Weygand JM, Ipavich FM, Wurz P, Paquette JA, Bochsler P (2001b) Determination of the Ar/Ca solar wind elemental abundance ratio using SOHO/CELIAS/MTOF. In: Wimmer-Schweingruber RF (ed) Solar and galactic composition. AIP conference proceedings, Melville, NY, vol CP-598, pp 101-106

Wiens RC, Bochsler P, Burnett DS, Wimmer-Schweingruber RF (2004) Solar and solar-wind isotopic compositions. Earth Planet Sci Lett 222:697-712

Wilhelm K, Curdt E, Marsch E, Schühle U, Lemaire P, Gabriel A, Vial JC, Grewing M, Huber MCE, Jordan SD, Poland AI, Thomas RJ, Kühne M, Timothy JG, Hassler DM, Siegmund OHW (1995) SUMER-solar ultraviolet measurements of emitted radiation. Solar Phys 162:189-231

Wimmer-Schweingruber RF (1994) Oxygen, helium, and hydrogen in the solar wind: SWICS/ULYSSES results. PhD thesis, University of Bern, Switzerland

Wimmer-Schweingruber RF, von Steiger R, Paerli R (1997) Solar wind stream interfaces in corrotating interaction regions. SWICS/Ulysses results. J Geophys Res 102:17,407-17,417

Wimmer-Schweingruber RF, Bochsler P, Kern O, Gloeckler G, Hamilton DC (1998) First determination of the silicon isotopic composition of the solar wind: WIND/MASS results. J Geophys Res 103(A9):20621-20630

Wimmer-Schweingruber RF, Bochsler P, Gloeckler G (2001) The isotopic composition of oxygen in the fast solar wind:ACE/SWIMS. Geophys Res Lett 28:2763-2766

Wurz P, Bochsler P, Paquette JA, Ipavich FM (2003) Calcium abundance in the solar wind. Astrophys J 583:489-495 